\title{
Soft Robotics in Minimally Invasive Surgery
}

\author{
Mark Runciman, ${ }^{1}$ Ara Darzi, ${ }^{2}$ and George P. Mylonas ${ }^{1}$
}

\begin{abstract}
Soft robotic devices have desirable traits for applications in minimally invasive surgery (MIS), but many interdisciplinary challenges remain unsolved. To understand current technologies, we carried out a keyword search using the Web of Science and Scopus databases, applied inclusion and exclusion criteria, and compared several characteristics of the soft robotic devices for MIS in the resulting articles. There was low diversity in the device designs and a wide-ranging level of detail regarding their capabilities. We propose a standardized comparison methodology to characterize soft robotics for various MIS applications, which will aid designers producing the next generation of devices.
\end{abstract}

Keywords: medical robotics, minimally invasive surgery, soft robots

\section{Introduction}

M INIMALLY INVASIVE SURGERY (MIS) involves the use of long rigid or flexible surgical instruments that are inserted into the body through small incisions or natural orifices, in contrast to open surgery where large incisions are used to access the target anatomy directly. The goal of MIS is to complete a surgical procedure as safely and quickly as possible, while minimizing damage to peripheral tissue. MIS is being used with increasing frequency as an alternative to open surgery because of the improvements it can bring to patient safety, cosmesis, recovery time, shorter hospital stay, fewer postoperative complications, and pain. ${ }^{1}$ This review details a literature search targeted at articles describing novel soft robotic devices for MIS.

Central to MIS is the field of endoscopy; the process of viewing the inside of the body by directly inserting an optical device into the area of interest. The optical device is called an endoscope and several different types exist. Today, an endoscope commonly refers to a long flexible tube approximately $1.5-2 \mathrm{~m}$ in length equipped with a high-resolution camera and a light source at its tip. The tip can be actively steered by means of two thumb-controlled dials at its proximal end. Typically, there are working channels along the endoscope's length to supply air and water, and through which small, flexible instruments can be introduced for performing basic therapeutic procedures. Flexible endoscopes of this description are used for visualization of the upper gastrointestinal (GI) tract (gastroscope) and lower GI tract (colonoscope). However, rigid endoscopes of varying length and diameter are also used in many applications, for example, to visualize the abdomen (laparoscope), brain, (neuroendoscope), joints (arthroscope), and esophagus (esophagoscope). There are a wide range of endoscopic procedures involving either diagnosis or therapy on many parts of the body. Flexible and rigid endoscopes can vary in diameter and length depending on the application and the patient.

Surgical tools allow surgeons to grasp, dissect, remove, and suture tissue inside the body. ${ }^{2}$ A common example of MIS is endoscopic surgery for abdominal procedures, where a laparoscope and two or three long, rigid surgical tools of typical diameter around $5 \mathrm{~mm}$ are introduced into the abdomen through multiple individual small incisions. Several approaches have been developed to make MIS even less invasive and to enable new procedures that are impossible with traditional open surgery. One of these improved approaches is single-incision laparoscopic surgery, which involves inserting not only a laparoscope but also two rigid instruments

\footnotetext{
${ }^{1}$ Human-Centred Automation, Robotics and Monitoring in Surgery (HARMS) Lab, Department of Surgery and Cancer, Imperial College London, London, United Kingdom.

${ }^{2}$ Department of Surgery and Cancer, Imperial College London, London, United Kingdom.

(C) Mark Runciman et al. 2019; Published by Mary Ann Liebert, Inc. This Open Access article is distributed under the terms of the Creative Commons License (http://creativecommons.org/licenses/by/4.0), which permits unrestricted use, distribution, and reproduction in any medium, provided the original work is properly cited.
} 
through a single larger incision in the abdomen, preferably at the umbilicus, therefore reducing the number of incisions, but increasing the difficulty of the procedure. Natural orifice transluminal endoscopic surgery (NOTES) is a technique in which the abdomen is accessed using a long, flexible endoscope inserted through the mouth, anus, or vagina, and offers the benefit of avoiding abdominal incisions entirely. ${ }^{3}$ Instead, NOTES is performed through internal incisions that allow the endoscope to cross between tubular structures within the body, known as lumen, to adjacent cavities. MIS can also be performed on the brain by removing part of the skull and placing a port, through which a neuroendoscope and surgical instruments are passed to gain access to target tissue deep in the brain. ${ }^{4}$

MIS is characterized by small, easily deformable, dynamically changing, and unstructured workspaces, poor visibility with few visual markers for orientation, and the use of long, narrow instruments. Long, rigid instruments used in some forms of MIS suffer from the fulcrum effect, caused by the point of insertion of the instrument into the body, acting as a point of rotation that inverts the surgeon's movements and can amplify hand tremor, making the instruments more difficult to use. ${ }^{5}$ In current robotic MIS approaches, a surgeon controls a rigid robotic device that in turn controls the motion of the modified surgical instruments. The forces exerted at the tip of manually operated laparoscopic instruments can range between 0.1 and $10 \mathrm{~N},{ }^{6}$ so designers of robotic systems aim to achieve similar performance. In addition, robotic systems deliver precision, stability, motion scaling, and other benefits, but are unable to navigate tortuous paths due to their inflexibility and sometimes their large size, meaning they cannot provide access to all target anatomy. Flexible endoscopes and instruments are therefore used when the surgical site cannot be reached by rigid devices and, if flexible devices would be ineffective, open surgery may be the only option. Robotic systems with multiple instruments are also affected by instrument clashing, ${ }^{7}$ which makes manipulating the instruments more complex due to their overlapping workspaces. Using some robotic systems can also present difficulties with changing instruments during a procedure.

Instruments that are difficult to use result in lengthy procedures and high risk of causing unwanted damage to the patient. ${ }^{8}$ Furthermore, years of training are sometimes required to become an expert in their use. Patient pain is often caused as a result of the instruments deforming or perforating the tissue surrounding them, which can be caused by using endoscopic instruments that are too stiff. ${ }^{9}$ Damage or pain to the patient can also be caused when using flexible devices, and an example of this is looping of the colon during colonoscopy. ${ }^{10}$ In addition, problems still remain with positioning, dexterity, force exertion, and visualization when using flexible instruments and endoscopes. ${ }^{11}$ Research into soft robotics aims to bring together the controllability of rigid robotics, the access capabilities of flexible instruments, and the safety of soft materials by solving these problems.

Soft robotics focuses on using soft, compliant materials to construct robotic devices. Due to the materials they are made from, soft robots are ideal for dealing with unstructured environments or interacting with humans because they can deform around their environment. ${ }^{12}$ This differs from the traditional robot design approach of using rigid materials for both robot links and joints and is very well suited to medical applications, where eliminating patient trauma and pain are highly important. ${ }^{13}$ The challenges faced in MIS make compliance, variable stiffness, and safety some of the most important design criteria, ${ }^{14}$ and the field of soft robotics is well placed to meet these demands. In the authors' experience, soft materials achieve high patient acceptability in comparison with robotic devices made from metallic or other rigid materials. A colon cancer patient representation group also found an unintimidating appearance to be more important than the footprint. Clinicians specializing in applications on the GI tract and who are familiar with its delicate mechanical properties also expressed a preference for soft devices that would be less likely to cause patient pain and trauma.

Unfortunately, there are many trade-offs in return for the increase to patient safety, including low force exertion, poor controllability, and a lack of sensing capabilities, as for example discussed in Hughes et al. ${ }^{15}$ Simulation of soft robots is difficult and computationally expensive because compliant materials exhibit nonlinear responses to strain and soft devices have many degrees of freedom, which hinders the ability to design soft robots predictably. ${ }^{16}$ Soft robots can achieve large changes in volume, shape, and stiffness, which are impossible for conventional ones and which give them a unique advantage. Harnessing these capabilities and tackling the problems with low force exertion and controllability will deliver the next-generation instruments for MIS.

An advantage of soft materials is that they are often economical, readily available, and easy to handle; the most common example being the large range of elastomers used in much of the current research. By using economical soft materials and new manufacturing techniques, it is also becoming possible to develop disposable, patient-specific, low-cost, and rapidly manufactured robotic devices for MIS. Being more affordable than traditional robotic systems, soft robotic devices have the potential to become widely available ${ }^{17}$ and this makes them a candidate for frugal design approaches that could have high impact. Further advancements in materials and manufacturing will confirm the potential for patientspecific soft robotics in the future. However, there may be additional regulatory requirements for customizable medical devices. Specifically, manufacturers must ensure that each customized device meets the appropriate quality and safety requirements; hence, reliable manufacture is highly important.

The objective of this review is to provide an overview of the soft robotic devices that are under development in the field of MIS so that designers may more easily identify new ways of overcoming the numerous challenges that are currently faced.

In the next section, the methodology used to carry out the literature search is reported, followed by a description of the comparison process and the results of the literature search. The application areas of the devices include endoscopic procedures for both diagnostic and therapeutic purposes. Throughout the rest of this review, the working principles, materials, manufacturing, actuation, variable stiffness, locomotion, and sensing methods found on the selected soft robotic devices are described in more detail. This will highlight the challenges across many disciplines that have to be considered and the lack of a standard method of comparison for soft robotic devices in MIS. 
Table 1. Keyword Search Terms and Results

\begin{tabular}{lccc}
\hline Database & \multicolumn{1}{c}{ Keyword search terms } & No. of results & Date \\
\hline Web of Science & $\begin{array}{c}\text { TS =("soft robot*”) AND TS=(MIS OR “minimal* invasiv*” } \\
\text { OR endoscop*) }\end{array}$ & 93 & $10 / 12 / 18$ \\
Scopus & $\begin{array}{c}\text { TITLE-ABS-KEY ("soft robot*”) AND (TITLE-ABS-KEY (mis OR } \\
\text { "minimal* invasiv*”) OR TITLE-ABS-KEY (endoscop*)) }\end{array}$ & 88 & $10 / 12 / 18$ \\
\hline
\end{tabular}

\section{Literature Search}

A keyword search was performed on Web of Science and Scopus for articles describing soft robotic devices designed for use in surgery or MIS procedures. The keyword searches used in the selected databases with the appropriate Boolean operators and syntax are summarized in Table 1 . The reference lists of these articles and other similar articles were also used as a source for a small proportion of additional search results. The inclusion and exclusion criteria described below were then applied and duplicate articles removed. This yielded 35 unique articles, each detailing a soft robotic device for use in minimally invasive applications.

\section{Inclusion criteria}

- The article described the design and/or manufacture of a robotic device.

- The device described was made of soft materials and relied on soft robotic principles.

- The main application for the device was surgery, MIS, endoscopy, laparoscopy, bronchoscopy, colonoscopy, or catheterization.

- The article was written in English.

- The article was accessible by the author at the time of the search.

\section{Exclusion criteria}

- The device described was not designed to be used in MIS procedures.

- The device was not compliant, or it contained too many rigid parts to be easily compressed.

There has been a growth in interest each year since around 2013, which can be seen by the number and cumulative total of results plotted in Figure 1. This was possibly influenced by the STIFF-FLOP surgical manipulator project ${ }^{18}$ that started in 2012 .

\section{Comparison Framework}

Of the soft robotic devices in the articles remaining at the end of the literature search procedure, the main characteristics were noted. These characteristics are summarized in Table 2. This was done to more easily compare distinct devices and highlight differences in the various categories. In the next section, the result of the device comparison process is shown.

\section{Results}

The articles yielded by the literature search were compared using the framework described in the previous section and the results can be found in Table 3 .

\section{Working principles}

There are many different ways to design robotics systems and this section describes the working principles of some of the soft robots that resulted from the literature search.

Continuum robots. Continuum robots are robotic devices whose bodies do not contain single rigid links or joints, but are able to bend continuously and can be considered to have an infinite number of joints. Octopus tentacles are a source of inspiration for soft continuum robots as they can achieve highly dexterous control, bending, and stiffening behavior, despite consisting of purely soft tissue. Many continuum robot designs are also modular or composed of several base units that can be controlled separately, as opposed to a single unit. Figure 2 shows some examples of different styles of continuum robot. Modularity can have benefits when

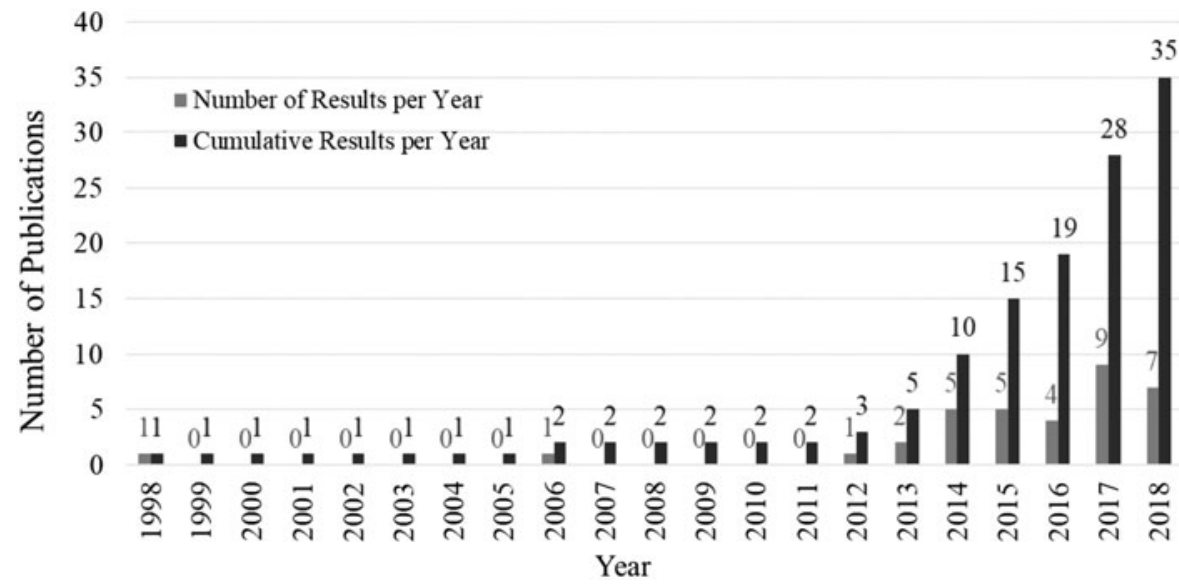

FIG. 1. Number of results and cumulative results per year. 
Table 2. Characteristics Used for Comparison Framework

\begin{tabular}{|c|c|}
\hline Characteristic & Description \\
\hline Application & $\begin{array}{l}\text { The application for which the device was designed. These include MIS, } \\
\text { which may require incision, and endoscopy, which uses natural orifices } \\
\text { and manipulation for devices that were designed to grip. }\end{array}$ \\
\hline Working Principle & $\begin{array}{l}\text { Fundamental structure of robot that was designed. Examples include the } \\
\text { following: continuum, serial, and peristaltic. }\end{array}$ \\
\hline Dimensions & Characteristic dimensions of the device. \\
\hline Materials & Materials used in the device construction focus on soft materials. \\
\hline Manufacturing Methods & $\begin{array}{l}\text { Main method of fabrication, whether it requires single or multiple } \\
\text { processes, manual or automatic assembly. }\end{array}$ \\
\hline Actuation Methods & All actuation and stiffening methods featured on the device. \\
\hline Locomotion & $\begin{array}{l}\text { Method of positioning the robot, whether it is manually or robotically } \\
\text { positioned or self-propelled. }\end{array}$ \\
\hline Sensors & All proprioceptive or diagnostic sensors on the device. \\
\hline Force exertion & Results of testing carried out on each device. Varies based on type of robot. \\
\hline Bending, twisting, elongation, expansion & The extent of each motion as appropriate. Varies based on type of robot. \\
\hline Variable Stiffness Mechanisms & $\begin{array}{l}\text { Describing the capability to control the stiffness. Varies based on type of } \\
\text { robot. }\end{array}$ \\
\hline Instrumentation/instrumentation channels & $\begin{array}{l}\text { Description of embedded instrumentation or number of channels available } \\
\text { for instruments. }\end{array}$ \\
\hline
\end{tabular}

MIS, minimally invasive surgery.

different parts of the robot are required to have different qualities, for example, the middle section of an arm is required to exert force or remain rigid, while the tip exhibits compliance. $^{45}$

Continuum robots seem to be an appropriate choice for MIS applications because they require only one entry point and can achieve large bending angles, permitting exploration of body cavities, which is impossible with rigid instruments. However, continuum robots also require a base or support structure and the ability to exert force diminishes along their length, which produces a trade-off between actuation and explorative capabilities. This style of robot was the most common among the 32 results of the literature search, with 18 single module continuum robots, ${ }^{19-34} 6$ modular continuum robots, ${ }^{38-43}$ and 2 robots capable of both peristaltic crawling and continuum motion. ${ }^{46,47}$

Peristaltic robots. Peristaltic robots are self-propelled devices that often take inspiration from the likes of earthworms, inchworms, and snakes. These three models differ in certain ways, but all of them depend on anisotropic friction to achieve locomotion. ${ }^{54}$ Only inchworm- and earthworm-style peristaltic motion were observed in the results of the literature review and they are described here.

In earthworms, the radii of several segments of its tubelike body are reduced at the same time as their lengths are increased, while other segments behind them expand their radii and shorten their length. This pattern of contraction/ elongation and expansion/shortening moves along the body of the worm in the opposite direction to its motion. The effect of this is to provide anchor points behind the elongating sections such that the friction in the forward direction is less than that in the backward direction. This form of peristaltic crawling was the most popular among the selfpropelling robots in the results, used in three out of four examples. ${ }^{51,53,55}$ The method in Suzumori et al. ${ }^{51}$ is slightly different from the others, as an elastomer sheath wrapped around a colonoscope was produced to help with scope advancement by generating travelling deformation waves, in contrast to the self-propelling colonoscope replacements in Connolly et al. ${ }^{53}$ and Takeshima and Takayama. ${ }^{55}$

The mechanism for inchworm motion is similar, except that anchor points are found only at the head and tail, while the body is contracted and elongated. An example of inchworm-style motion was implemented in a self-propelled endoscope that makes use of a cable-driven actuation mechanism, ${ }^{46}$ which anchors its head or tail by pressing into the colon wall, and then advances by releasing cable tension in the center of its body, which elongates using passive spring action.

Soft peristaltic robots provide the potential to provide access without stretching the colon and causing damage or pain to the patient as current techniques do. Furthermore, selfpropelling devices offer a step toward the automation of routine procedures. Effortlessly advancing through the colon would allow more attention to be devoted to diagnosis.

Serial robots. Serial robots consist of several prismatic or rotational joints that are coupled together by links, often to form the well-known robot arm structure. This type of robot is mentioned in this study because it is usually associated with traditional robotics constructed from rigid materials; however, a soft serial robot consisting of pneumatic stiffened links and pneumatically actuated joints has been developed for safe interactions between people and industrial robots, achieving positioning error of $<1 \mathrm{~mm} .^{56}$ Soft pneumatic joints have also been developed, which can produce both linear and rotational motion for use in grippers, robotic arms and even mobile robots. ${ }^{57}$ In relation to medical applications, a pneumatically actuated serial mechanism was attached to the end of an endoscope in Russo et al. ${ }^{58}$ This mechanism integrates soft fluidic actuators into a rigid resin structure. Some grippers rely on serial mechanisms, such as that described in Pacchierotti et al. ${ }^{50}$ where straight, but soft fingers on a miniature starfish-like robot are actuated at a soft joint. 


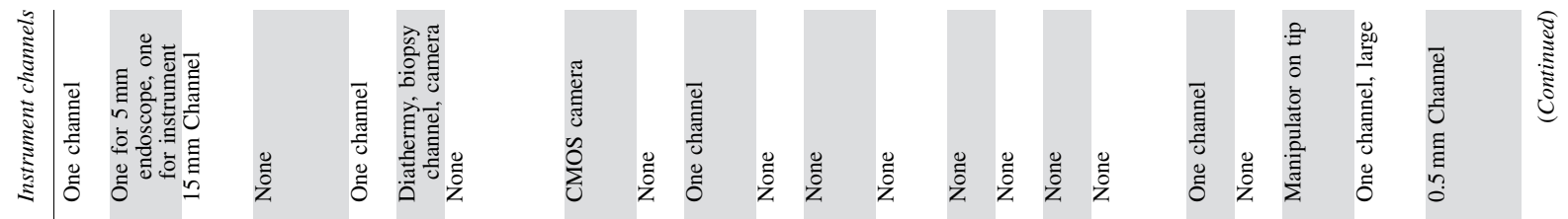
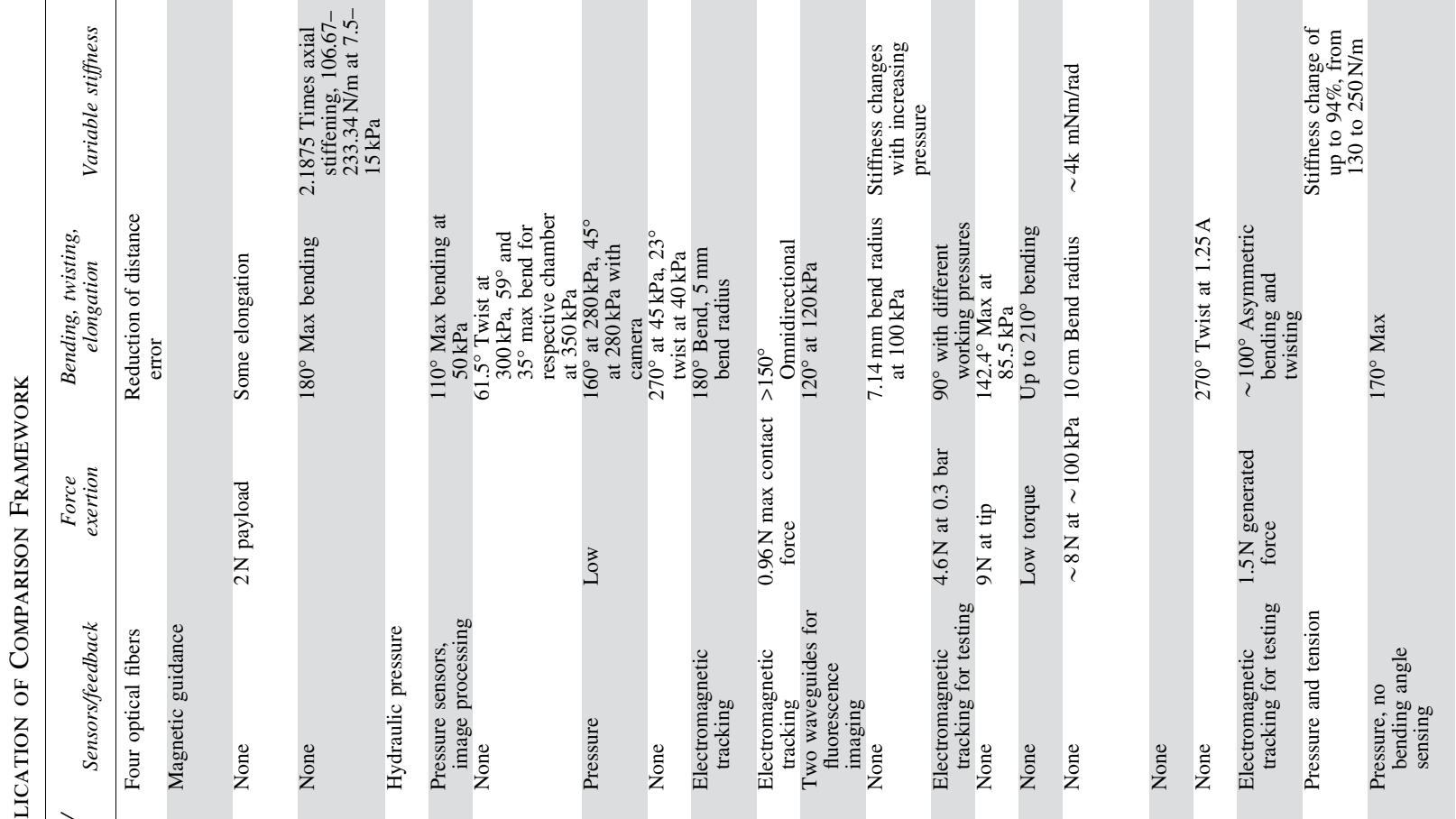

完突离

MIII1111111111111

|l|

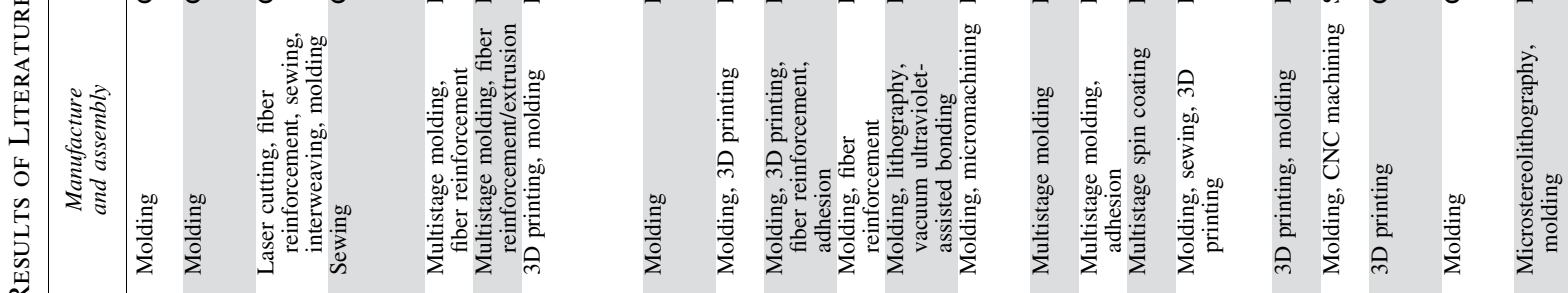

"III

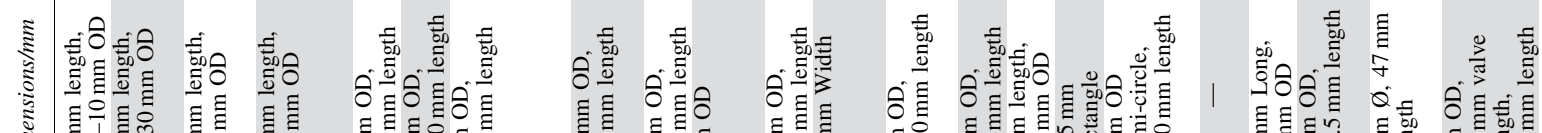

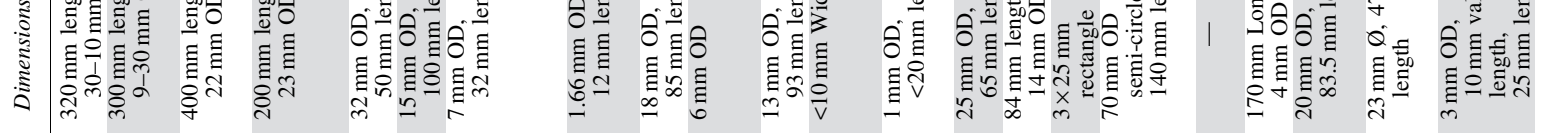

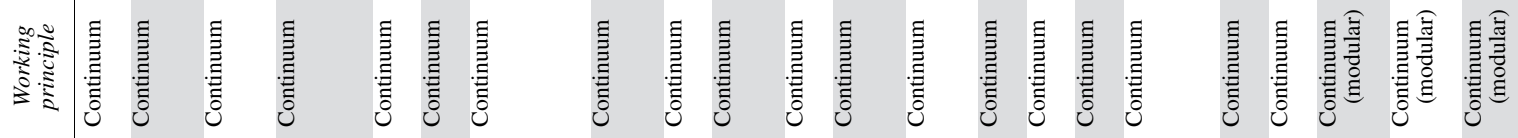

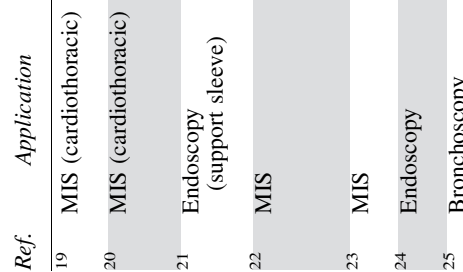

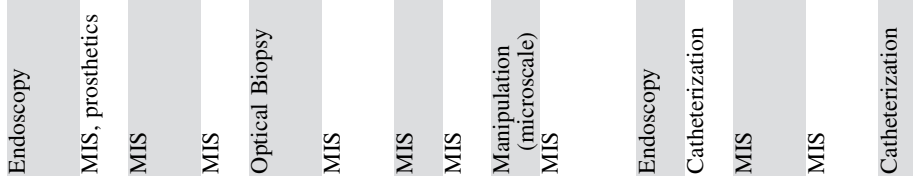




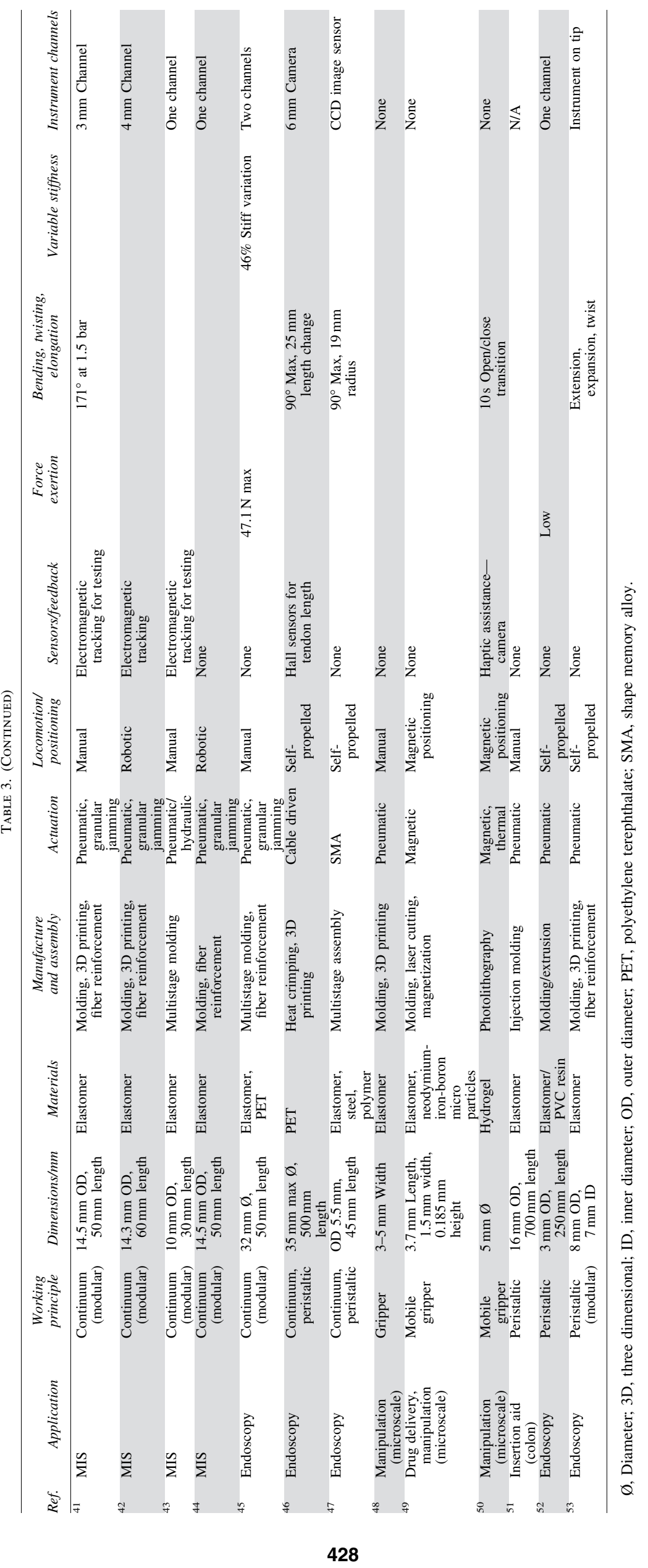




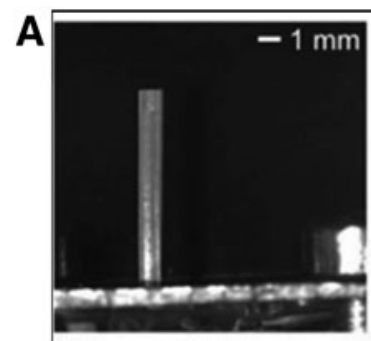

$0 \mathrm{MPa}$

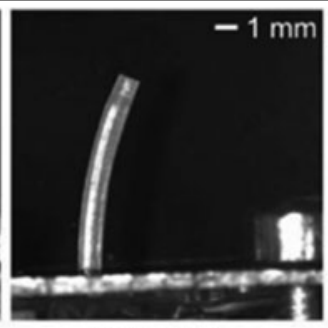

$0.10 \mathrm{MPa}$

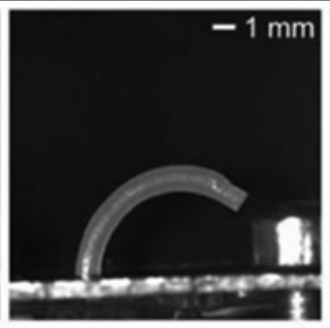

$0.20 \mathrm{MPa}$
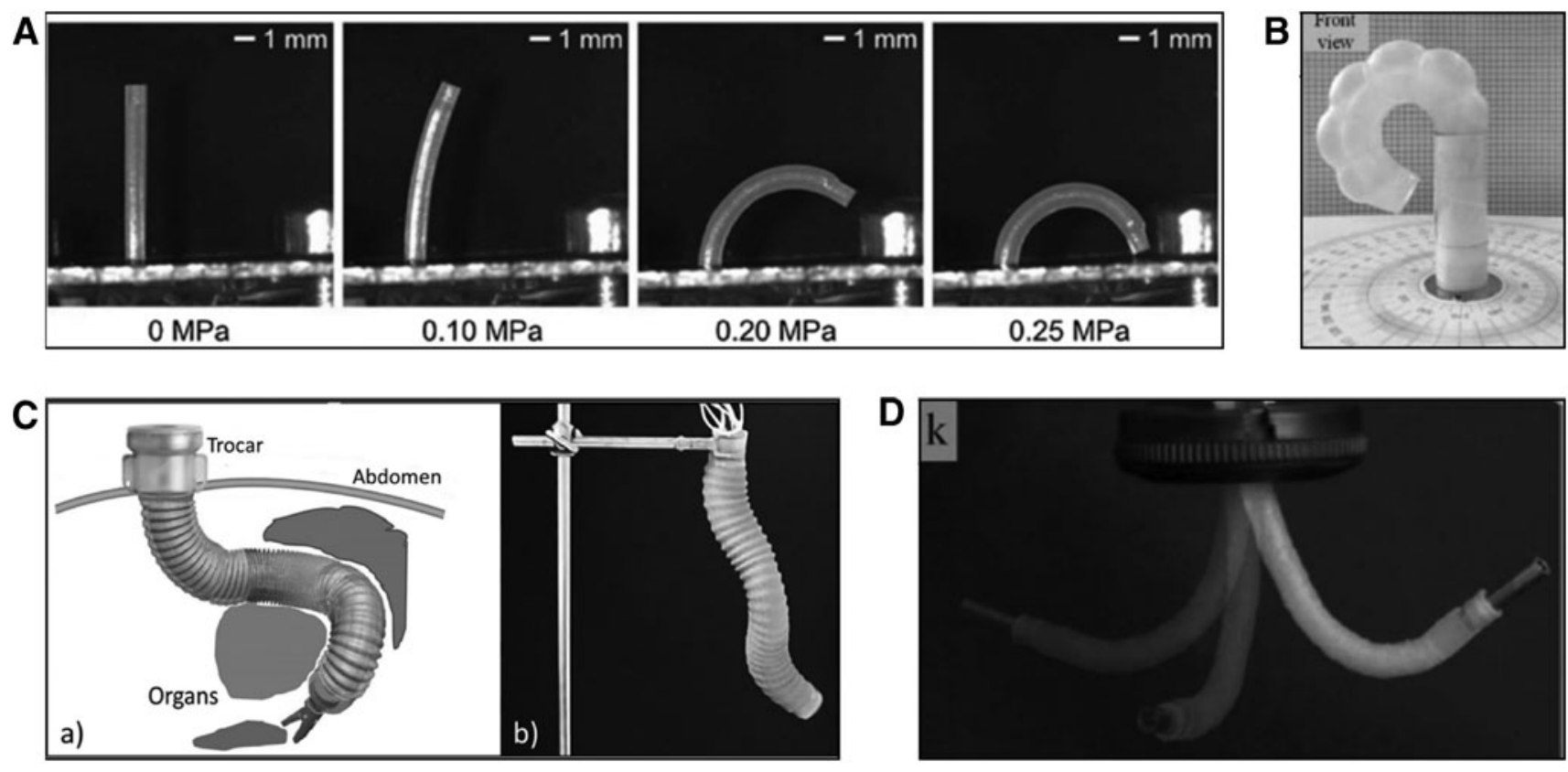

FIG. 2. Examples of continuum robots for MIS: (A) a single-chamber pneumatic microactuator, ${ }^{31}(\mathbf{B})$ a three-chambered pneumatic module, ${ }^{27}$ (C) the three-module STIFF-FLOP manipulator, ${ }^{88}$ (D) a miniature three-chambered manipulator with outer diameter of $6 \mathrm{~mm} .{ }^{28}$ MIS, minimally invasive surgery. Figure panels were reprinted with permission from their respective references.

A limitation of soft serial-type robots is that serial robots rely on discrete links to transfer force, so using deformable materials or variable stiffness mechanisms leads to low force exertion or large size. Furthermore, accurate control is achieved in traditional robots that use rigid components because the link lengths, joint rotations, and joint extensions are known or can be easily measured, whereas the same cannot be said for their soft counterparts. Their low controllability may be the main reason that no soft robot arms for MIS were observed in the literature search, coupled with the fact that the manufacture of a soft robot with several discrete links and joints would be more complex than the manufacture of a continuum robot. ${ }^{1}$

Hyper-redundant serial robots are composed of a large number of joints and have similar benefits to continuum robots, but also suffer similar trade-offs. Serial robots with high degrees of freedom (DOFs) are capable of assuming complex poses that could allow them to navigate tortuous paths and inspect complex shaped objects; however, force exertion diminishes with increasing length. In this way, they are apparently suitable to MIS applications, however, no articles describing hyper-redundant soft serial robots were yielded by the literature search. Complex manufacture and control appear to be the main challenges to face, but this may be an interesting target for research if appropriate manufacturing methods are developed.

\section{Materials}

Soft materials provide a valuable resource for designing atraumatic medical devices for use within the body because they can deform before causing damage. Soft materials include elastomers, such as polydimethylsiloxane (PDMS) and polyurethane; thin layers of plastics, such as polyethylene
(PE), polyethylene terephthalate (PET), or polypropylene (PP); and even metals that exhibit superelasticity and that have been manufactured to have very low cross-sectional area, such as Nickel Titanium ( $\mathrm{NiTi}$, Nitinol). Elastomers were used in 31 devices in the results of the literature review, plastics in 8, latex in 2 devices, and hydrogel and leather used in just one each. Three devices used NiTi wire as an actuator. One of the benefits of using elastomers and plastics in particular is their low cost and ready availability. As a result of using soft materials, it is likely that a soft device is magnetic resonance imaging compatible, which designers can capitalize on.

In some cases, it was unclear whether a device should be included in this review based on the rigidity of the materials used. For example, the continuum robots designed in $\mathrm{Li}$ and $\mathrm{Du}^{59}$ and Li et al. ${ }^{60}$ rely upon a series of vertebrae as the main support structure, which makes them highly flexible until some bending limit is reached and the vertebrae lock together. These may at first appear to be soft robots as they use soft materials and use actuation mechanisms commonly seen in the soft robotics field, such as cable-driven mechanisms. However, not all of the materials throughout the devices exhibit compliance and, therefore, still pose the threat of causing unwanted harm within the body due to their rigidity. Compliance, $k^{-1}$, is related to the length, $L$, elastic modulus, $E$, and the crosssectional area, $A$, of a material, as shown in Equation (1).

$$
k^{-1}=\frac{L}{E \cdot A}
$$

A device that is constructed from materials whose elastic moduli are orders of magnitude greater than human tissues can still therefore be classified as a soft robot if the materials' cross-sectional areas are small. ${ }^{61}$ Many elastomers are highly compliant due to their low Young's moduli, making them a 
popular choice for interaction with the patient in noninvasive medical applications.

A robot's stiffness, force exertion, and bending/twisting/ elongation/expansion can all be dependent on the elastomer used because the deformable bodies of elastomer-based robots often contain the chambers used for fluidic actuation. Extra care must therefore be taken when selecting elastomeric materials, due to their influence on many aspects of a device's functionality.

The development of soft analogues for more complex, currently rigid mechanisms is another challenge. Given that fluidic actuation is a popular actuation method, soft valves are of particular interest. Soft valves inspired by the three-flap, cone-shaped mitral valve in the heart were manufactured by silicone molding in Gilbertson et al., ${ }^{62}$ as an enabling technology for an entirely soft, self-propelling catheter.
Compliant valves that controlled flow by using the electrorheological principle were three-dimensional (3D) printed in Zatopa et al., ${ }^{63}$ using liquid metal eutectic gallium-indiumtin as the electrodes. The hydraulic valve could withstand over $250 \mathrm{kPa}$ of pressure when activated at a voltage of $5 \mathrm{kV}$, but this varied when the valve was put under strain. This is just one example of how using soft materials bring new challenges that have not been encountered with traditional materials; hence, making soft replacements for traditionally rigid components remains a challenge.

\section{Manufacturing methods}

There are a variety of manufacturing methods that can be used to make soft robotic devices. A brief summary of the most common methods is made in this section, followed by

Table 4. Manufacturing Process and Their Advantages and Disadvantages

\begin{tabular}{lll}
\hline Manufacture Process & \multicolumn{1}{c}{ Description } & \multicolumn{1}{c}{ Advantages } \\
\hline Molding & $\begin{array}{c}\text { A mold is manufactured and } \\
\text { filled with elastomer that is } \\
\text { left to cure. }\end{array}$ & $\begin{array}{l}\text { Simple process for simple } \\
\text { parts; molds can be } \\
\text { manufactured cheaply and } \\
\end{array}$ \\
& $\begin{array}{l}\text { relatively quickly, often } \\
\text { using additive } \\
\text { manufacturing; repeatable } \\
\text { (based on mold }\end{array}$ \\
& manufacture); expandable.
\end{tabular}

Extrusion

3D printing
elastomers

Shape deposition

Soft lithography

Micro stereo lithography

Spin coating
Material is forced through a die to produce a given cross section.

Devices are printed one layer at a time using thermoplastic elastomer or with a curing process.

Multiple molds and steps are used to mold varying materials in complex shapes.

Begin with bulk soft material substrate and either remove or deposit new material where the substrate has been exposed to radiation.

A series of cross-sectional layers are built up one at a time by radiating material at the base.

A substrate is rapidly rotated after application of a coating fluid, depositing a thin film on the substrate after a given time due to the centrifugal effect.
Large-scale production; long parts are possible; repeatable; forms internal lumen.

Highly adaptable; repeatable; expandable; range of design sizes.

Allows for embedding of components into a part; can combine different materials; allows convex features.

Very small feature sizes possible, down to $\sim 500 \mu \mathrm{m}$; repeatable process.

Repeatable process; adaptable.

Possible to produce very thin films; quick process; repeatable

\section{Disadvantages}

Limited material selection; defects such as bubbles can form in the elastomer body; often requires additional parts/materials to reinforce or prevent large deformations; lower limit on size; lower limit on feature size; adding additional features can make manufacture complex.

High equipment costs; crosssection is constant along the length; cross-sectional dies are not easily adapted; feature size limited to.

Support material required, can limit size as material deforms under own weight; time-consuming.

Multistep process; timeconsuming.

Upper limit on device size; time consuming; difficult to incorporate additional functionality onto small devices.

Upper limit on device size; difficult to incorporate additional functionality onto small devices.

Multistage process, punctuated by curing processes and actuation fluid channel placement; upper limit on size; difficult to incorporate additional functionality onto small devices. 
an analysis of the manufacturing methods that were used to produce the devices yielded in the keyword search. Novel manufacturing methods will play a key role in developing patient-specific devices.

Time, cost, repeatability, reliability, adaptability, expandability, quality, size, feature size, equipment, and compatible materials are all factors that need to be considered when choosing manufacturing methods. Another of the challenges with patient-specific devices is to ensure the manufacturing quality of each individual device. Making sure the performance, reliability, and safety of multiple units that have each been adapted for particular patients and particular medical operations present a substantial hurdle using current approaches. Furthermore, the ability to demonstrate the safety of all variations of a general device design within a certain range of dimensions, applications, and target anatomy may also present issues with certification, which will also have to develop in conjunction with the patient-specific surgical technology that will be seen in the near future.

Manufacturing approaches using rigid plastics have been developed, which aim to make patient-specific, disposable devices feasible in terms of manufacture time and cost by using additive manufacturing. ${ }^{64,65}$ However, there are still many limitations to producing robotic devices made of soft materials quickly and cheaply, while maintaining the high level of performance necessary in MIS applications. Although the materials used in elastomer molding are economical, the time required for manually carrying out additional manufacturing processes (such as fiber reinforcement, CNC machining, or 3D printing) and additional assembly (such as adhesion, placement of a sheath, or connection of multiple modules) often hinders rapid manufacture. High manufacturing times or high costs, due to complex procedures, equipment, or materials, prohibit the development of disposable, singleuse, or patient-specific devices. A summary of manufacturing processes that can be used to make soft robotic devices is found in Table 4.

Molding of elastomeric polymers was the most popular manufacturing method, possibly because it is a simple process, including the fact that automated processes can be relied

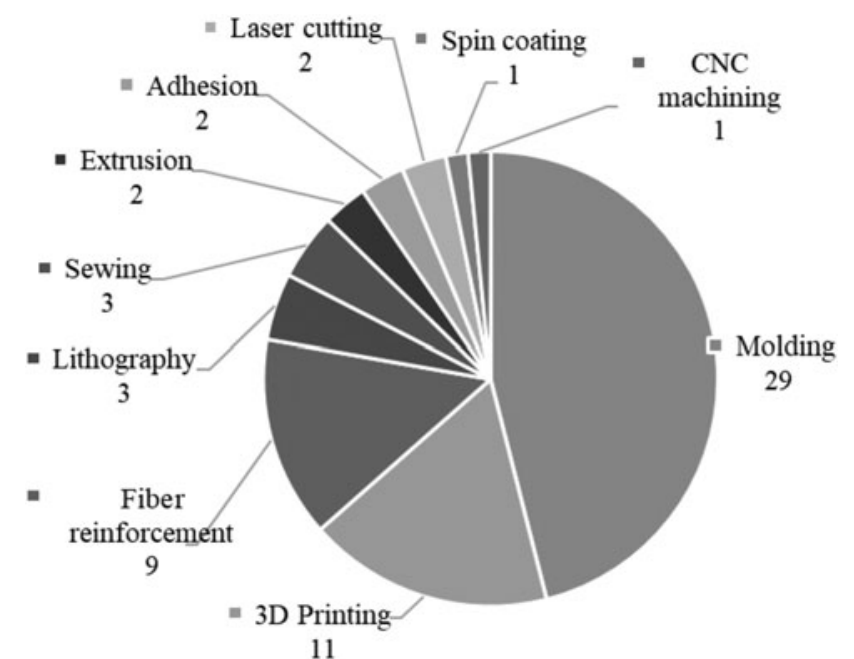

FIG. 3. Number of uses of different manufacturing techniques among the selected devices at the focus of this review. More than one technique could be used per device. upon for fabrication of molds. Figure 3 shows the number of instances where different manufacturing methods were used to make the devices from the literature search, where multiple processes could be used on one device. In most cases, a 3D printer was used to produce the mold, although molds have been made from many materials, including laser cut Plexiglas, ${ }^{43} 3 \mathrm{D}$ printed plastics, and CNC milled metals. The elastomeric materials that can be used with molding processes are typically soft, making them desirable in minimally invasive applications.

Manufacturing methods such as molding, extrusion, and shape deposition prevent adaptability due to the necessity to newly fabricate or assemble not only the constituent parts but also auxiliary parts that are used in the manufacturing steps, for example, molds or dies that need to be changed to produce a new shape. In contrast, devices can be more readily adapted when they rely on rapid manufacturing techniques that are very easily controlled, taking advantage of computer-assisted design and automated processes, for example additive manufacturing or photolithography.

Difficulties with molding elastomers include bubbles forming in the material before it sets, giving rise to structural inconsistencies. Manufacturing methods were the main problem in the fabrication of a low-cost elastomer endoscope. $^{24}$ The difficulty of demolding larger or more complex shapes can result in damage to the molded part and may require more complex molds. It has also been reported that achieving even thicknesses can be difficult, which is one of the issues caused by using 3D printed molds, as well as the ability to produce small enough molds and prevent them from melting during heating to cure the elastomer. ${ }^{25}$

Often, multistage processes are used that can be timeconsuming and can introduce errors. These additional steps may include the following: fiber reinforcement, filling with granular particles, inserting layer jamming material, adhesion, sewing, heat crimping, or heat sealing. The review in Gorissen et $a .^{66}$ is very thorough in detailing the manufacture of pneumatic elastomer actuators and reports that bonding or sealing elastic materials to create an inflatable chamber frequently leads to actuator failure. Figure 4 highlights a number of manufacturing methods and some of the steps involved.

Using an extrusion process, a helical braided tube device design was made to be adaptable, in that new devices could be made specifically for given pipe diameters. This particular design, however, encountered difficulty propelling itself through pipes with large changes in diameter. ${ }^{55}$ Despite an easily alterable design, the device itself was unable to deal with an adaptable environment, making it unsuitable for MIS applications.

A microscale soft actuator was manufactured by using the spin coating technique to produce a pneumatic channel within multiple layers of PDMS material. ${ }^{34}$ Bending motion was produced upon pressurization due to the asymmetric cross-section of the actuator, which was controlled by precisely adjusting the layer thicknesses of the PDMS. Furthermore, the bending behavior was altered by cutting the profile of the actuator in different ways, for, example, in a trapezium with the pneumatic channel located centrally, or in a rectangular shape with the pneumatic channel placed parallel to the sides at varying distances or at an angle to the sides. These various profiles and channel placements produced a variety of bending behaviors, including curling, spiraling, and bidirectional spiraling. 

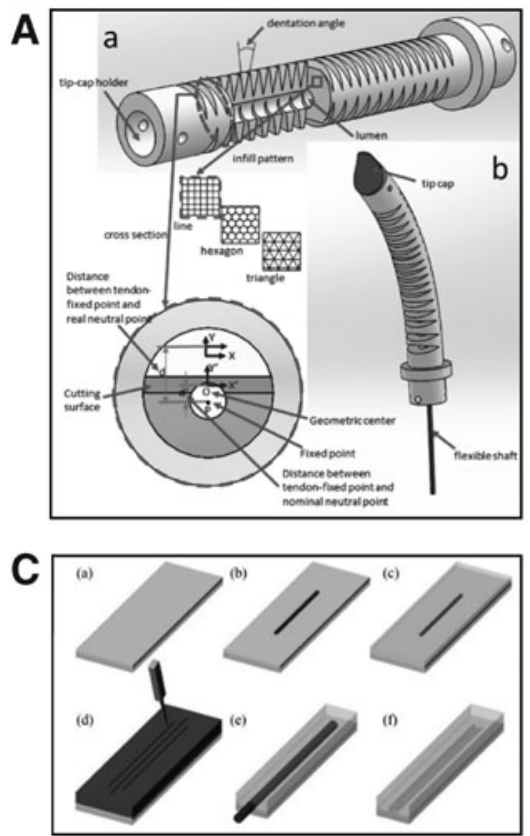
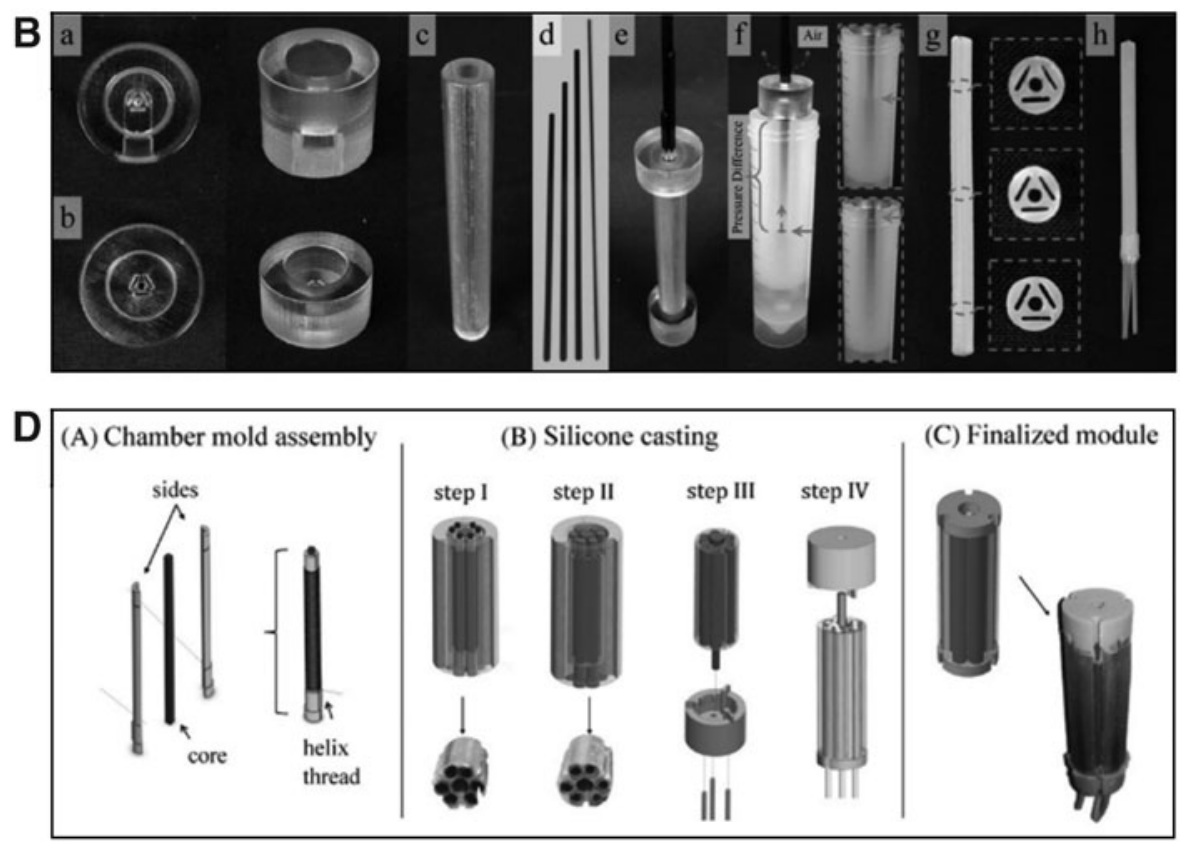

FIG. 4. Examples of different manufacturing techniques: (A) 3D printing of a continuum manipulator using thermoplastic polyurethane, with different possible infill patterns ${ }^{38}(\mathbf{B})$ stages of an "inverse flow injection" molding method, ${ }^{28}(\mathbf{C})$ a microactuator manufactured by multistage spin coating, ${ }^{34}$ (D) the steps followed to manufacture a STIFF-FLOP manipulator. $^{41} 3 \mathrm{D}$, three dimensional. Figure panels were reprinted with permission from their respective references.

Mechanical programming/embodied intelligence. How a device or component moves is based on its mechanical construction; hence, the designer can alter the behavior of a device by subtly changing how it is manufactured, while maintaining the underlying core design. The area of soft robotics is ripe for this design methodology because behavior during actuation is dependent on the body of the device in many cases. Therefore, there is opportunity to use their construction to influence their deformation. Figure 5 features a selection of soft robotic devices that use this approach.

As an example, the deformation of a pressurized elastomeric fluidic actuator can be constrained by wrapping the actuator in reinforcing fiber. A variety of bending, twisting, expansion, and elongation behavior can then be produced depending on the fiber winding angle without changing the elastomeric actuator. This concept was explored in detail in Connolly et al., ${ }^{67}$ where a self-propelling soft device was manufactured by chaining fiber-reinforced pneumatic actuators in series that were identical, except for the fiber winding angle and the number of fiber layers. Three different fiber winding patterns were used, which resulted in extension, expansion, or twisting of the actuators when pressurized, so a robot capable of peristalsis and with a twisting end effector was produced by connecting and pressurizing the actuators in a specific sequence. This shows how the motion of a simple pneumatic element can be changed by processing it in different ways.

Pairs of finger-like pneumatic actuators of semicircular profile with fiber reinforcement at distinct angles have been coupled together in parallel back to back to produce both bending and twisting motions; however, the bending behavior is asymmetric as a result of the asymmetric design. ${ }^{25}$ Again, by changing the fiber winding angle, the motion of the manipulator can be programmed.
A cable-driven continuum robot was manufactured by 3D printing using thermoplastic elastomer ${ }^{38}$ and the mechanical behavior of the device was shown to change with its crosssectional shape. Devices with triangular, semicircle, and rectangular cross-sections were manufactured and it was found that the device with triangular cross-section could transmit less force that the other versions. This also demonstrates how using an automated manufacturing process facilitates design adaptations. Various cross-sectional shapes of the actuation chambers, chamber length to module length ratios, and chamber proximity to the outer wall were tested in a pneumatically driven elastomeric continuum device, ${ }^{32}$ where both the pressure required to achieve a $90^{\circ}$ bend and the ballooning area were measured. This information could be used to design a manipulator with specific bending behavior.

Pneumatic chambers can be formed by heat-sealing multiple layers of flexible planar materials and complex, actuating structures can be produced by controlling the heatsealed patterns. ${ }^{68}$ Using this method, the bending angles of pneumatic hinges at a given working pressure were programmed by changing the aspect ratio of the heat-sealed, diamond-shaped pattern.

\section{Actuation, stiffness variation, and locomotion methods}

In this section, three important aspects of soft robotics are discussed: actuation, variable stiffness, and locomotion. These are highlighted because they can instill robotic devices with many of the abilities necessary in medical applications, for example, manipulating objects, supporting loads, and propelling themselves. Advances in actuation, variable stiffness, and locomotion will enable robotic devices that increase surgeon dexterity, access, and the ability to intuitively use their skills as if they were performing an open surgery. 

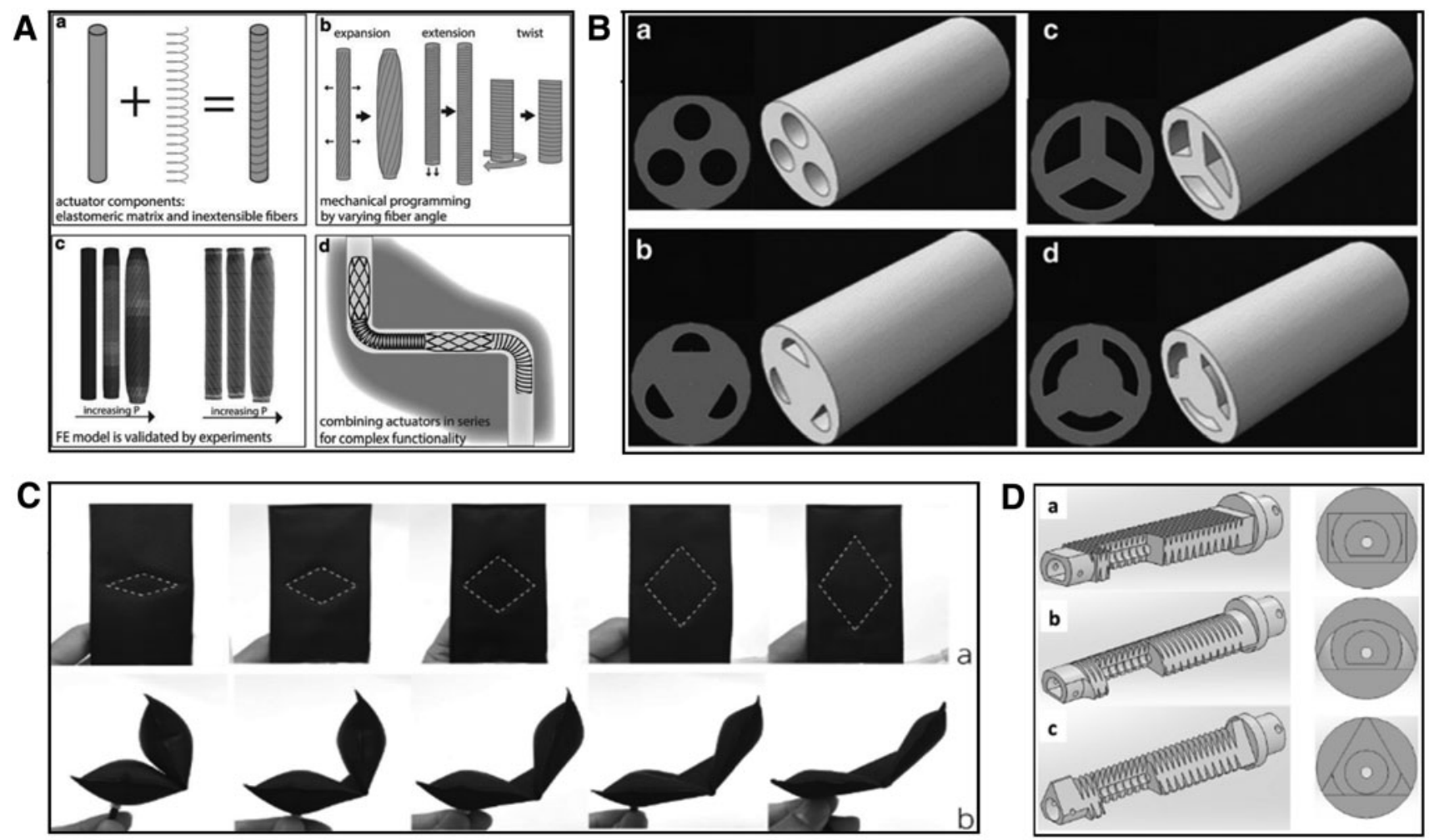

FIG. 5. Mechanical programming achieved by varying device design or manufacture: (A) a pneumatic actuator made to expand, extend, or twist by changing its fiber winding angle, ${ }^{67}$ (B) four variations of a continuum robot body whose inner chambers determine its behavior, ${ }^{32}$ (C) different bending angles achieved at a given operating pressure by changing the aspect ratio of a diamond hinge pattern, ${ }^{68}$ (D) various cross-sectional shapes of 3D printed manipulators change their mechanical behavior. ${ }^{38}$ Figure panels were reprinted with permission from their respective references.

Actuation methods. Actuation methods produce motion of a robotic device, permitting bending, elongation, and force exertion, for example. These technologies, appropriately controlled, will give soft robotic devices the dexterity to allow surgeons to perform surgical tasks more easily. Table 5 briefly summarizes actuation methods that were used in the articles resulting from the keyword search, commonly used in soft material robotics.

The most common actuation method used among the devices from the literature search was pneumatic actuation, followed by cable-driven, hydraulic, shape memory alloy (SMA) and thermal actuation. Electroactive polymers (EAPs) are at the center of much research; however, no device using EAPs was present in the results of this literature search. The lack of "dry" EAPs may be attributed to the high voltages that are required for actuation, ${ }^{69}$ and lack of "wet" EAPs may be because only low forces can be exerted and they have long response times. ${ }^{70}$ Figure 6 shows the number of times different methods were used among the results, where multiple actuation techniques could be employed in a single device. The following section describes some examples of the different actuation methods.

Fluidic actuation blurs the lines between actuation and variable stiffness because the internal pressure of a chamber influences the stiffness and will do so in different ways for different actuation fluids. When deformations in the crosssection of a beam occur, this can cause an increase in stiffness due to Poisson's effect. ${ }^{31}$ The coupling of actuation and stiffening effects is a problem for the most popular soft ro- botic actuation method because the stiffness varies with the pose, making control difficult.

Most devices that use pneumatic actuation are manufactured by molding elastomeric materials and most are continuum robots. Many of these require strain-limiting layers to avoid ballooning. If larger diameter/stronger/stiffer devices are required, then the central channel may be unavailable for instrumentation because it may disturb the pneumatic actuation channel. If a continuum robot is manufactured with instrumentation channels, making it a more useful device in a surgical context, then the trade-off is lower force exertion and larger size because space for actuation chambers is reduced. For highly elastic pneumatically actuated designs, the crosssectional area, actuation chamber length and wall thickness can change the bending behavior. ${ }^{32}$ Hence, it can be challenging to introduce flexible cameras and instrumentation channels that would normally be found on a traditional endoscope without altering the controllability, the size, or both.

The load-bearing limits of a cable-driven system can be increased by increasing the diameter of the cable, which will not significantly change the size of the device. However, to exert larger forces, the support structure for the cables must be stronger. This may consequently impact on the materials used or the size of the resulting device. Cable- and SMAdriven systems depend upon the structure that they are built into, while fluidic actuators depend upon the materials that constitute the fluid chambers.

There are also safety issues that need to be confronted when using each of these actuation methods, given the 
Table 5. Summary of Actuation Methods

\begin{tabular}{|c|c|c|c|}
\hline Actuation method & Description & Advantages & Disadvantages \\
\hline Pneumatic & $\begin{array}{l}\text { Vary pressure of } \\
\text { gas within a } \\
\text { chamber }\end{array}$ & $\begin{array}{l}\text { Capable of producing a range of } \\
\text { movements, for example, } \\
\text { elongation, expansion, twisting, and } \\
\text { bending, plus stiffening; large } \\
\text { volume changes are possible; quick } \\
\text { response; very lightweight; good } \\
\text { power density. }\end{array}$ & $\begin{array}{l}\text { High pressures needed to exert larger } \\
\text { forces; nonlinear response makes } \\
\text { control more complex; depends on } \\
\text { fluid flow that introduces a delay; } \\
\text { risk of puncture or gas escape that } \\
\text { could cause damage/harm. }\end{array}$ \\
\hline Hydraulic & $\begin{array}{l}\text { Vary pressure of } \\
\text { liquid within a } \\
\text { chamber }\end{array}$ & $\begin{array}{l}\text { Capable of exerting large forces; } \\
\text { incompressible actuation fluid } \\
\text { makes precise control more } \\
\text { achievable; high power density. }\end{array}$ & $\begin{array}{l}\text { Adds weight to device; slower } \\
\text { response time; depends on fluid flow } \\
\text { that introduces a delay; jets of } \\
\text { pressurized liquid could damage soft } \\
\text { tissue. }\end{array}$ \\
\hline Cable driven & $\begin{array}{l}\text { Change tension or } \\
\text { length of cable } \\
\text { to exert force }\end{array}$ & $\begin{array}{l}\text { Capable of exerting larger forces; fast } \\
\text { response; small form factor; } \\
\text { lightweight. }\end{array}$ & $\begin{array}{l}\text { Only capable of actuating in one } \\
\text { direction, when cable is in tension } \\
\text { (zero compression stiffness); friction } \\
\text { forces can become large in long } \\
\text { cables along tortuous paths. }\end{array}$ \\
\hline SMA & $\begin{array}{l}\text { Deform alloy by } \\
\text { changing its } \\
\text { temperature }\end{array}$ & $\begin{array}{l}\text { Easily controlled; medium velocity } \\
\text { response. }\end{array}$ & $\begin{array}{l}\text { Sensitive to environment; potential } \\
\text { safety risk posed by thermal } \\
\text { dissipation; hysteresis error. }\end{array}$ \\
\hline
\end{tabular}

sensitive surroundings and the possibility of the surgical instruments interacting with the soft robot itself. The risk of piercing a pressurized chamber can be mitigated by utilizing a protective sheath; however, this may increase size or manufacturing complexity. Another factor to take into account is that the temperature reached by some SMA actuators can exceed the working temperatures of some elastomers. ${ }^{37}$ There are also safety issues related to exposing soft tissue to higher temperatures.

The length of SMA wires affects their mechanical behavior. In Shim et al., ${ }^{37}$ the length of the NiTi SMA wire influenced both the maximum twisting angle and the maximum twisting torque. Similarly, the size of fluidic actuators influences their force exertion capabilities and their rigidity. These examples highlight the trade-off between size and power.

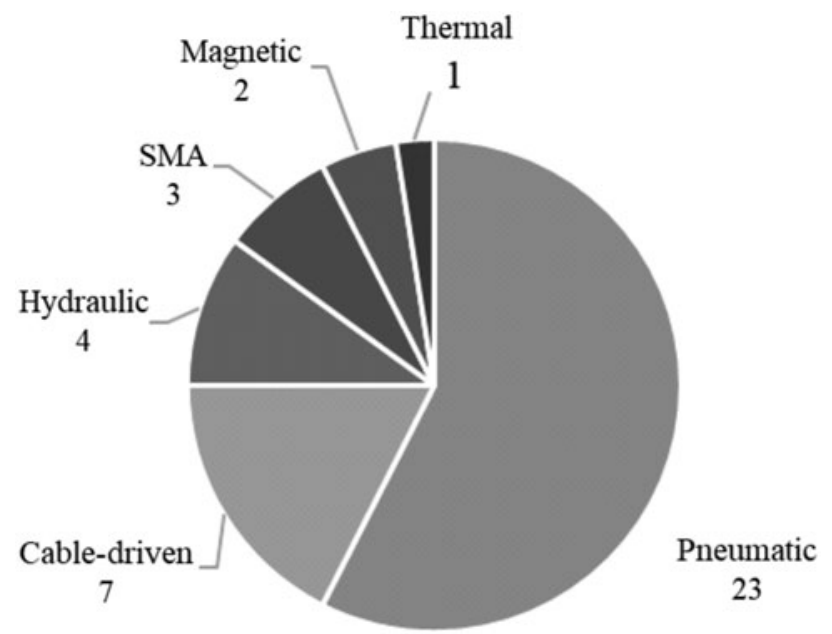

FIG. 6. The number of devices from the literature search that used each actuation method, with more than one actuation method being used in some cases.
Variable stiffness mechanisms. Only granular/particle and layer jamming offer variable stiffness without producing motion, as opposed to actuation methods that can not only cause changes in stiffness but also exert force, for example, cable-driven or pneumatic systems. Granular and layer jamming are compared in Table 6 . For a full review of variable stiffness mechanisms used in medical devices, the reader is directed to Blanc et al. ${ }^{71}$

Jamming relies upon locking together the particles of granular materials or several layers of planar materials, which can be induced when pressure is exerted on these materials in enclosed chambers. Figure 7 displays some interesting implementations of jamming techniques. Jamming based on negative gauge pressure is limited by the ambient pressure surrounding the jamming chamber, but can also be achieved by applying a positive gauge pressure to the jamming material. In $\mathrm{Li}$ et al., ${ }^{35}$ jamming with positive pressure is achieved by constraining a finger-like elastomer device consisting of both an expanding pneumatically actuated chamber and a jamming chamber filled with $2 \mathrm{~mm}$ diameter glass beads within an inextensible leather sheath. Inflating the pneumatic chamber causes it to expand radially, pressing the jamming chamber against the outer sheath and locking the glass beads together to increase stiffness. This design increases in stiffness as it bends and uses only one actively actuated chamber; therefore, it is a useful configuration in applications where the number of input tubes or cables linking a device to its power source needs to be minimized, or the design needs to be as simple or robust as possible. In general, a high number of jamming layers, high actuation pressure, and high coefficient of friction of the laminar material are recommended to achieve the highest stiffness change using layer jamming. ${ }^{72}$

Several actuation methods can be used together to produce an increase in stiffness, for example, pneumatic actuation and a cable-driven system as in Stilli et al. $^{22}$ and Shiva et al. ${ }^{39}$ Actuation and variable stiffness mechanisms can be used together, for example, pneumatic actuation and granular 
Table 6. Summary of Stiffening Mechanisms Used in Articles from Literature Search

\begin{tabular}{|c|c|c|c|}
\hline Stiffening method & Description & Advantages & Disadvantages \\
\hline $\begin{array}{l}\text { Granular/particle } \\
\text { jamming }\end{array}$ & $\begin{array}{l}\text { Force particles of a granular } \\
\text { solid together to stiffen using } \\
\text { particle locking }\end{array}$ & $\begin{array}{l}\text { Can achieve large variation in } \\
\text { stiffness; shape locking }\end{array}$ & $\begin{array}{l}\text { Adds weight and volume to the } \\
\text { design; underlying physical } \\
\text { relations unclear, difficult to } \\
\text { model; particles can spread } \\
\text { unevenly, leading to } \\
\text { nonuniform stiffness }\end{array}$ \\
\hline Layer jamming & $\begin{array}{l}\text { Force layers of a planar material } \\
\text { together to stiffen using } \\
\text { interlayer friction }\end{array}$ & $\begin{array}{l}\text { Low profile; capable of shape } \\
\text { locking; even stiffness if } \\
\text { layers are well constrained } \\
\text { relative to each other }\end{array}$ & $\begin{array}{l}\text { Adds rigidity in inactivated state; } \\
\text { limited increase in rigidity }\end{array}$ \\
\hline
\end{tabular}

jamming as in Li et al., ${ }^{35}$ Diodato et al., ${ }^{44}$ and Cianchetti et al $^{45}$ or a cable-driven system used together with a layer jamming mechanism as in Kim et al. ${ }^{21}$

The combination of variable stiffness mechanisms and soft, flexible materials has been used to alter a robot's kinematics online, something that is impossible with traditional, rigid robots. A two-finger, elastomeric cable-driven gripper featuring three serially connected layer jamming modules per finger was manufactured in Narang et al. ${ }^{72}$ and was able to move each finger either as a continuum mechanism, when the modules were unjammed, or as a serial mechanism with two joints between the three jammed links.
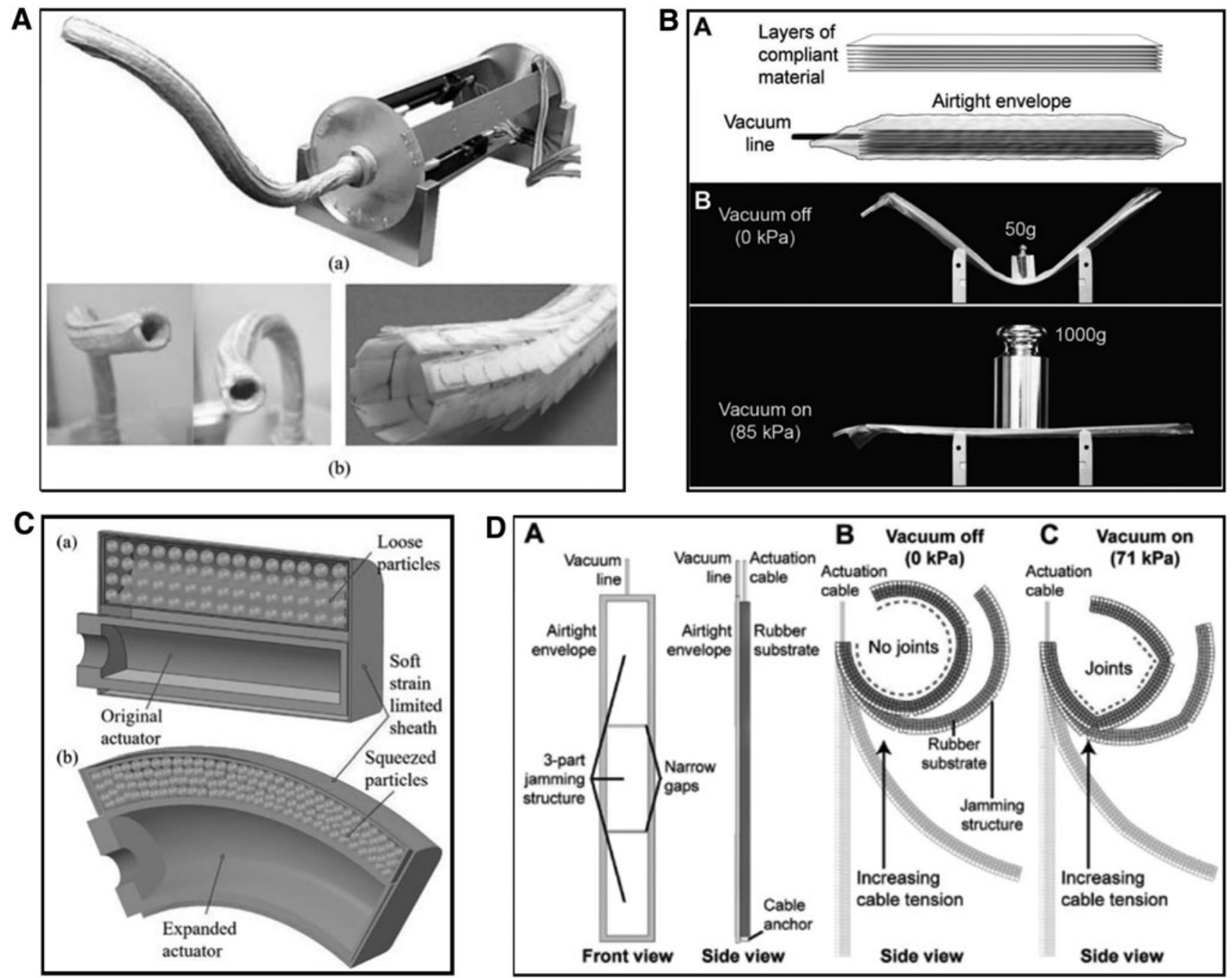

FIG. 7. Granular and layer jamming techniques: (A) layer jamming employed in a stiffening sleeve for use with endoscopic devices, ${ }^{21}$ (B) the layer jamming principle, variable stiffness can be achieved by applying a vacuum to a sealed chamber, ${ }^{72}$ (C) a passive particle jamming method to simultaneously actuate and stiffen a device, ${ }^{35}$ (D) layer jamming in a cable actuated finger used to alter its structure online. ${ }^{72}$ Figure panels were reprinted with permission from their respective references. 
Locomotion. Robotic locomotion in the context of medical devices should aim to enable access to areas that are difficult to reach and do so without causing patient discomfort. At present, the most common method of delivering a robotic device to the target tissue is by manual insertion, which is a problem for devices made of nonrigid materials that will buckle under loading, and also for those constructed from rigid materials that are difficult to pass through the tortuous paths of lumen within the body without stretching tissue and causing pain. Another method is robotic guidance, where a soft robotic device may be attached to another robotic device, typically a rigid device, which controls the soft device's positioning. ${ }^{20,44}$ Magnetic fields can also be controlled to precisely position micro robots that could be used for drug delivery, ${ }^{49,50}$ although the hardware needed to achieve this can be bulky. Finally, self-propelling devices use on-board mechanisms to interact directly with their sur- roundings to exert thrust forces. Peristaltic crawling was the only self-propulsion method developed in the results of the literature search. Figure 8 highlights a number of locomotion methods revealed by the literature search. For an in-depth analysis of soft robotic locomotion, the review ${ }^{54}$ is a valuable resource. Table 7 briefly summarizes two types of peristaltic crawling, inspired by the earthworm and inchworm, respectively.

Figure 9 shows the reliance of the devices yielded in the literature search on the method of manually placing the device, a method that plays a large role in causing patient pain. Patient discomfort is one of the main issues with colonoscopies currently, ${ }^{73}$ so a reliable and painless selfpropulsion method would be of great value to the field.

The method proposed in Bernth et al. ${ }^{46}$ shows the ability to self-propel using a two-anchor inchworm method; however, it relies upon anchoring at a single point on the colon wall
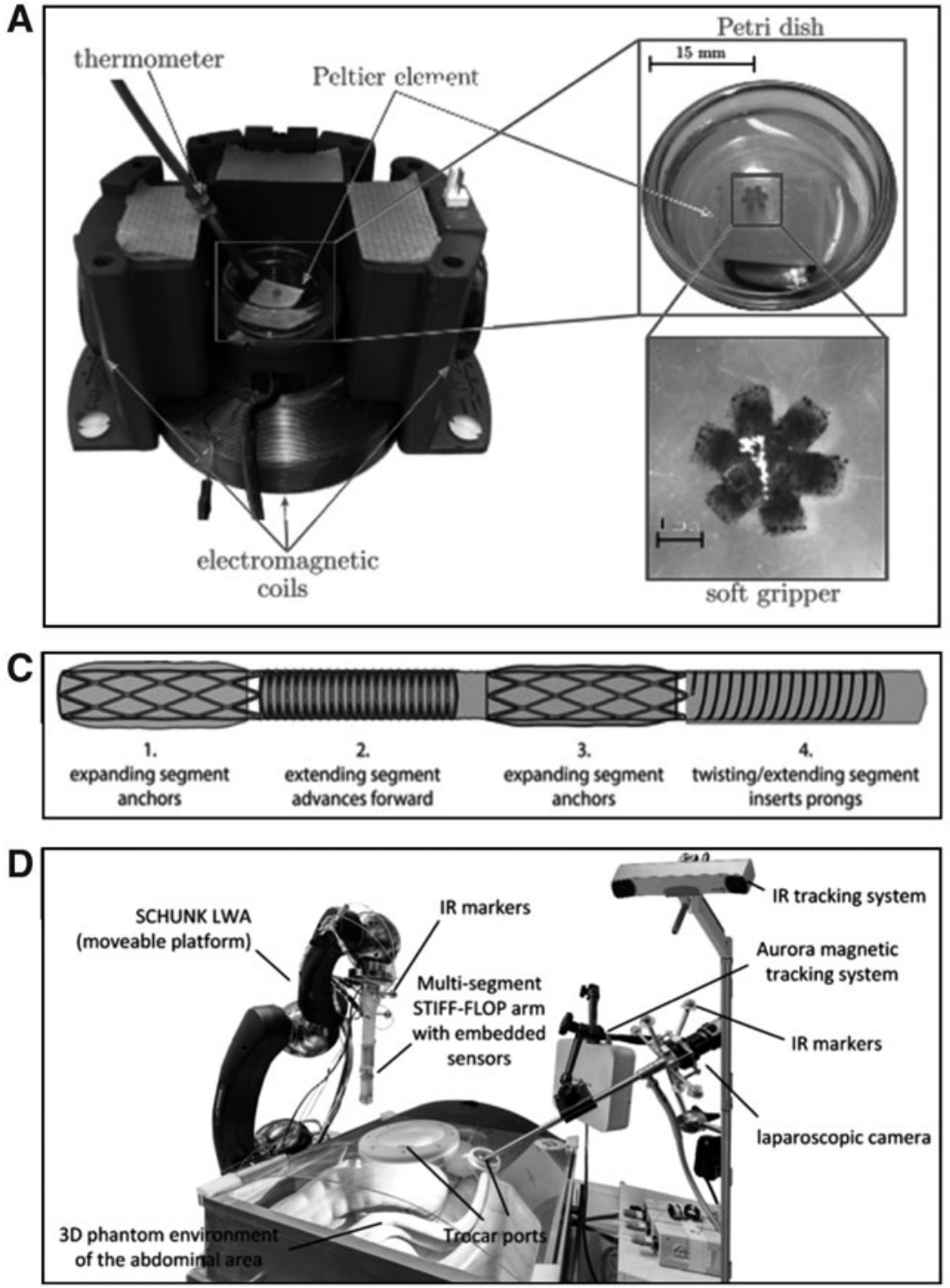
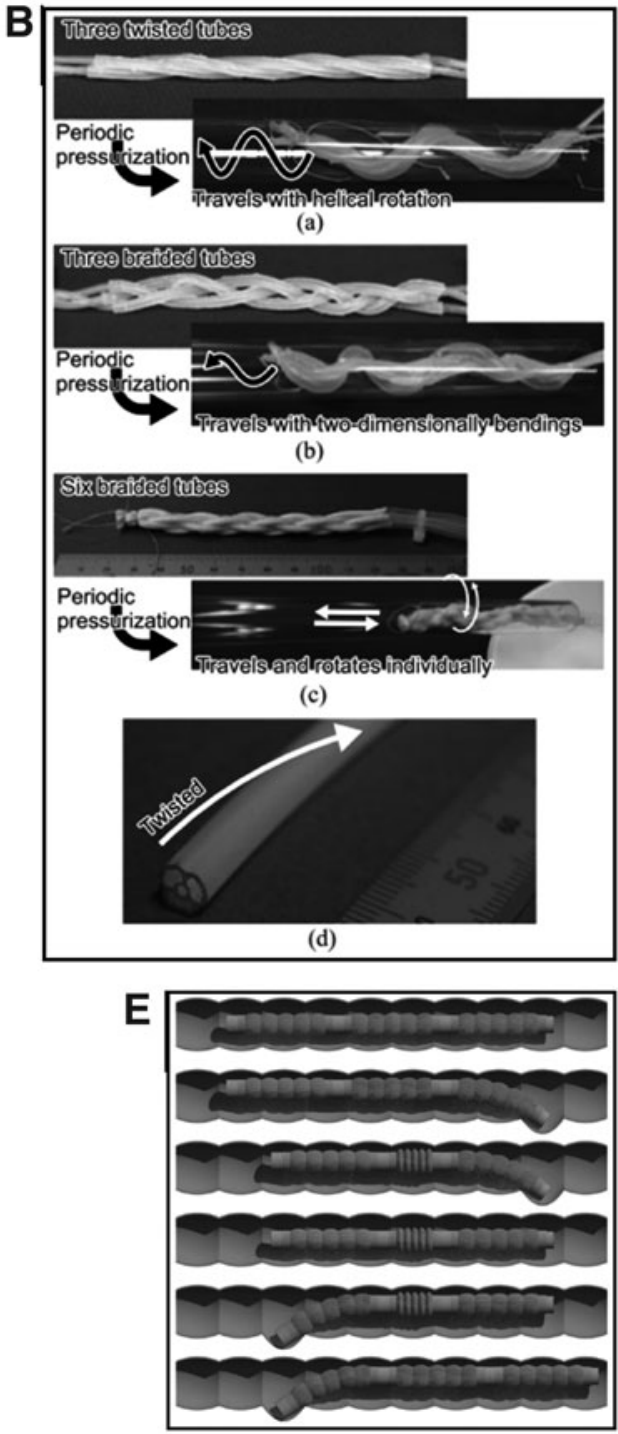

FIG. 8. Locomotion methods: (A) magnetic fields used to maneuver a microgripper on a Petri dish, ${ }^{50}$ (B) braided actuator bundles achieving both peristaltic forward motion and twisting in different configurations, ${ }^{55}$ (C) multiple pneumatic actuators each used for different parts of a peristaltic locomotion cycle, ${ }^{67}$ (D) a robotically guided STIFF-FLOP manipulator, $^{42}(\mathbf{E})$ visualization of an inchworm-style crawling robot anchoring on the inner colon wall by applying a force. ${ }^{46}$ Figure panels were reprinted with permission from their respective references. 
Table 7. Summary of Peristaltic Crawling Types

\begin{tabular}{|c|c|c|c|}
\hline Locomotion method & Description & Advantages & Disadvantages \\
\hline $\begin{array}{l}\text { Earthworm-inspired } \\
\text { peristalsis (wave) }\end{array}$ & $\begin{array}{l}\text { Waves of anchoring and } \\
\text { elongation pass along } \\
\text { whole body }\end{array}$ & $\begin{array}{l}\text { Higher friction may be } \\
\text { generated by a greater } \\
\text { number of anchoring } \\
\text { sections; radial force } \\
\text { exertion, may prevent } \\
\text { looping. }\end{array}$ & $\begin{array}{l}\text { Multiple actuation units to be } \\
\text { sequentially controlled } \\
\text { increases controller size } \\
\text { and complexity; no existing } \\
\text { sensors for speed or } \\
\text { position measurement. }\end{array}$ \\
\hline $\begin{array}{l}\text { Inchworm-inspired } \\
\text { peristalsis (two } \\
\text { anchor) }\end{array}$ & $\begin{array}{l}\text { The head and tail are } \\
\text { sequentially anchored, } \\
\text { while the body elongates } \\
\text { and contracts }\end{array}$ & $\begin{array}{l}\text { Simple sequential control is } \\
\text { possible; radial force } \\
\text { exertion, may prevent } \\
\text { looping; shorter body than } \\
\text { earthworm style. }\end{array}$ & $\begin{array}{l}\text { Movement is jerky; no } \\
\text { existing sensors for speed } \\
\text { or position measurement. }\end{array}$ \\
\hline
\end{tabular}

each cycle and this still may cause stretching and pain to the patient. Another way may be to anchor the device across a larger area so as to distribute the load, and this could be achieved by using more compliant materials that would deform to the inner colon wall.

A specifically designed anchoring mechanism was developed on the design of an endoscope attachment in Stopforth et al. ${ }^{24}$ with the goal of realizing autonomous exploration of the colon. The hydraulically powered endoscope probe could align itself with the center of a tube, and an image processing system designed to find the center of the colon was developed.

The existing soft self-propulsion mechanisms of the devices in the literature search are still slow. The highest speed of the peristaltic crawling device in Bernth et al. ${ }^{46}$ is $1.21 \mathrm{~mm} / \mathrm{s}$, with the authors stating it could travel from one end of a human colon measuring $1850 \mathrm{~mm}^{74}$ to the other in $\sim 30 \mathrm{~min}$. It takes between $20 \mathrm{~min}$ and $1 \mathrm{~h}$ to complete a standard colonoscopy, ${ }^{75}$ so the device would offer no improvement on procedure time. The friction force in the anchored state of Bernth et al. ${ }^{46}$ was $2 \mathrm{~N}$.

Using a different approach, three designs of three or six elastomer pneumatic tubes, either braided or twisted together, were developed in Takeshima and Takayama. ${ }^{55}$ The twisted three-tube device moved through a pipe with a helical

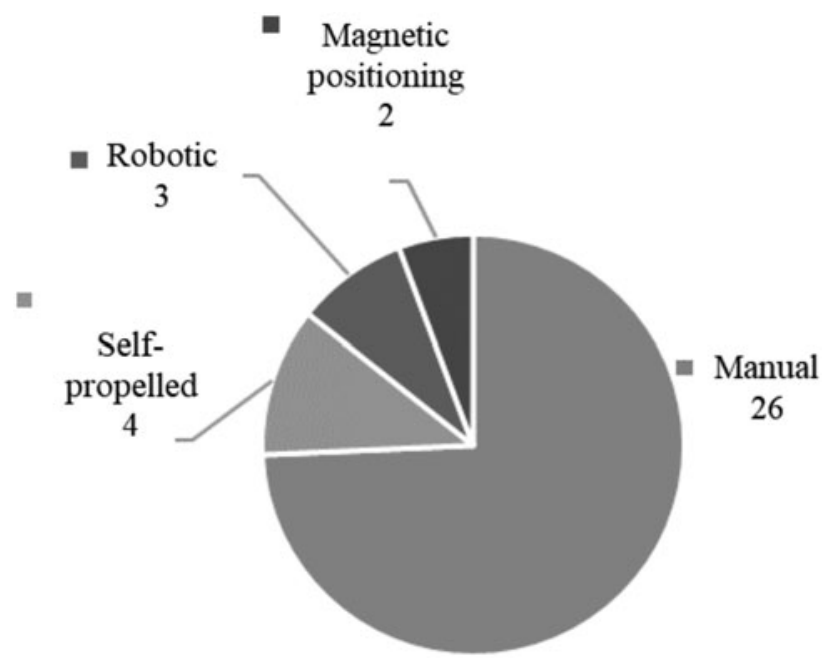

FIG. 9. Locomotion methods of devices from the literature search. trajectory, the braided three-tube device moved side to side in a serpentine manner, while the braided six-tube design could translate and rotate independently about the axis of the pipe. However, none of these were successful moving in pipes that had changes in diameter. A trade-off between locomotion capabilities and size must be confronted with peristaltic designs, as there, the physical extension of the device is relied upon. For example, the forward thrust force of the device in Takeshima and Takayama ${ }^{55}$ is dependent on its length. For applications within the lower GI tract, the problem of lack of traction in the unsupported, elastic colon still has no definitive answer.

A greater focus on locomotion methods for soft robotic devices in the lumen of the body will also enable NOTES, further reducing recovery times and eliminating visible scars. A problem with NOTES in terms of design of a selfpropelling device is that a change in locomotion method will likely be required as the device transitions between tubular structures such as the GI tract to the less constrained abdominal cavity. However, soft material robots are capable of changing their structure, volume, and working principle, among other qualities, so they are an appropriate candidate technology for solving this issue.

\section{Sensors}

This section describes different categories of sensors and how they might be used to provide useful information in the context of MIS. For a review of soft electronics, the reader is directed to Dickey. ${ }^{76}$

To gain meaningful knowledge about a robotic device, the environment that surrounds it, and the interaction between the two, measurements from a variety of sources must be taken. Sensors can be grouped into two main categories: proprioceptive and exteroceptive, which, respectively, measure characteristics of the robot itself and characteristics of its surroundings. Sensors of both types are important for control strategies and, in the MIS context, for preventing the robot from causing harm to the patient, for example, by exerting large forces on soft tissue or cutting off blood supply through prolonged pressure exertion and causing ischemia. Diagnostic sensors also gather information, not pertaining to robotic devices, but rather to provide valuable medical information about the patient.

Often the information gathered by one category of sensor is useless without information from another, so fusion of 
information from proprioceptive, exteroceptive, and diagnostic sensors is often required. In many cases, it is the output from diagnostic sensors that must be coupled with either proprioceptive or exteroceptive data to deliver useful information. A simple example could be a device that can detect early signs of cancer in the GI tract, which is made much more useful by also being able to accurately establish where the device, and therefore the cancer is located, for subsequent retargeting. Figure 10 displays some approaches to proprioceptive, exteroceptive, and diagnostic sensing with soft robots that are discussed further in this study.

Difficulty modeling soft robotic devices is a substantial problem. For sensor data to be used appropriately in a range of applications like control, haptic feedback, or diagnostics, a model of the robot in question is required. This poses a great challenge in the field of soft robotics because of the nonlinear behavior of many soft materials and actuation methods. ${ }^{77}$ Therefore, extra consideration must be given to sensor design, placement, and data interpretation. Furthermore, for shape changing soft robots that can reversibly transition between different kinematic regimes, numerous models may be needed to fully describe them. For example, the number and location of bending angle sensors on a continuum robot will differ from that of a serial robot, and the information the sensors provide needs to be contextualized appropriately in each case.

Empirical performance modeling was necessary in Takeshima and Takayama ${ }^{55}$ due to the difficulty of computationally modeling the braided three-chamber pneumatic device that had been developed. This highlights the need for less expensive computational modeling of soft robotic devices to achieve the aim of developing more accurate control systems without the need for experimental characterization of each individual device, which would inhibit customizable, patient-specific robots, for example.

Proprioceptive sensors. In the results of the literature search, there were more examples of proprioceptive sensors than exteroceptive or diagnostic sensors. Proprioceptive sensors are often used for control of robotic mechanisms because they can be used to estimate the pose or shape of the device, hence their high priority. There are a broad range of sensors that can be used for proprioception of soft robotic devices that do not significantly increase the stiffness, and they vary depending on the working principles or actuation methods that are being employed. Robots that are actuated using fluids may include pressure sensors, ${ }^{23,24,39,40}$ although there is often difficulty relating internal pressure to bending angle, especially in the presence of external forces on the device.

Cable-driven robots have used measurements of cable tension $^{39}$ and length, for example, using Hall effect sensors, ${ }^{46}$ but effects such as cable friction can affect these results. Robots made of elastomeric materials may use strain gauges to estimate the shape of the device, although none of the devices in the literature search used these. The field of soft strain sensors is well established because of their biomedical
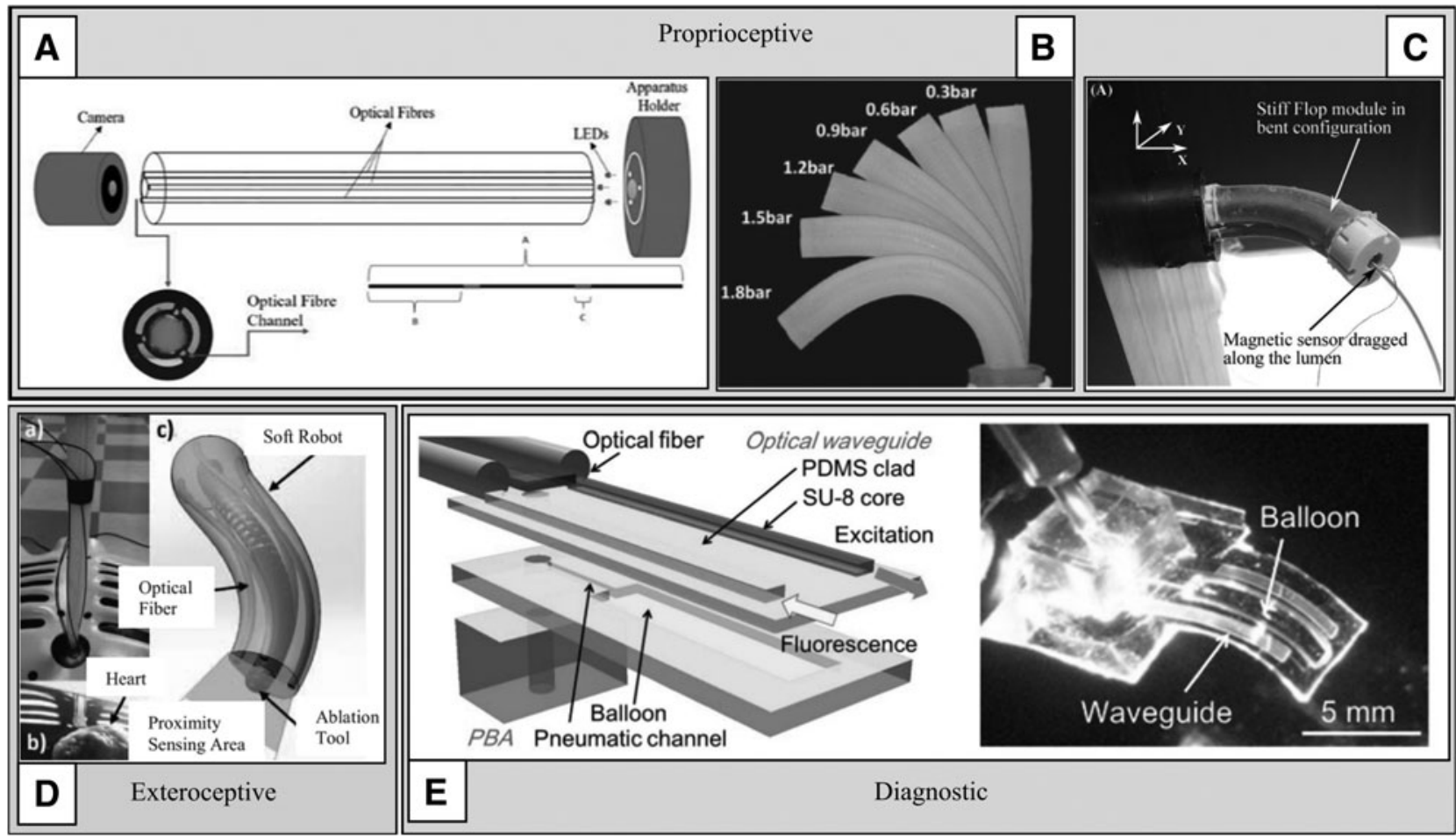

FIG. 10. Proprioceptive, exteroceptive, and diagnostic sensors in soft robots: (A) bending angle estimation using light intensity transmitted by optical fibers, ${ }^{79}$ (B) using interior air pressure to estimate angle, ${ }^{29}$ (C) using an EM tracker to record the pose of a STIFF-FLOP manipulator, ${ }^{4}$ (D) optical fibers for proximity sensing integrated into a cable-driven cardiac ablation tool, ${ }^{19}$ (E) an optical waveguide for fluorescence imaging integrated into a miniature elastomer actuator. ${ }^{30}$ EM, electromagnetic. Figure panels were reprinted with permission from their respective references. 
and wearable applications, and Amjadi et $a l^{78}$ is a useful review on the subject.

Optical fibers have been used with soft continuum robots to sense their bending angle. In $\mathrm{Al}$ Jaber and Althoefer ${ }^{79}$ three optical fibers were placed in a deformable rubber cylinder at $120^{\circ}$ to each other to estimate the curvature. A camera was attached to the end of the cylinder to detect the light transmitted through the optical fibers, emitted by RGB LEDs. By monitoring the intensity of the light in the camera feed, as well as the size, orientation, and position of the optical fiber tips, the bending angle could be estimated with an error between $2.3 \%$ and $17.6 \%$. This method is promising; however, it requires bulky hardware that would alter the mechanical behavior of a soft robotic device. Fiber Bragg Gratings (FBGs) embedded in optical fibers were integrated into a cable-driven soft continuum manipulator for the purpose of shape sensing in Wang et al. ${ }^{80}$ Despite showing good performance for simple robot configurations, it showed sensitivity to temperature change, inaccuracies in molding manufacture affecting optical fiber placement, and insensitivity to large deflections over short lengths of the soft robot.

Electromagnetic (EM) sensors have been used to track the tip of continuum robots, ${ }^{29,32,38,43}$ but these measurements can be affected by stray EM fields from other equipment ${ }^{81}$ or field distortion in the presence of metallic structures. Using continuum soft robots equipped with EM sensors, machine learning has been used to control tip position along given trajectories, which in each case improved the robots' abilities to follow a path. ${ }^{29,82}$ It would be valuable to see results of this approach using other sensor inputs that do not suffer from the same issues as EM trackers.

Imaging methods such as fluoroscopy and ultrasound can be used for shape sensing; however, fluoroscopy requires costly, bulky equipment and exposure of the patient to radiation, while ultrasound offers only low resolution. ${ }^{81}$

A novel additive manufacturing method has been developed to simultaneously print elastomer and liquid metal in a helical structure to form an inductive sensor that is sensitive to tensile and bending deformation. ${ }^{83}$ This flexible helical sheath is designed to be placed around a soft, cylindrical device such that the bending angle of the device and the sensor is the same. Deformations in the sensor then cause changes in inductance of the liquid metal core, which in this case is galinstan, an alloy of gallium, indium, and tin. Using this sensor, the bending radius of an endoscope was estimated in distinct poses with an error of between $1.53 \%$ and $7.96 \%$. Using liquid metals or other fluids as sensors permits highly flexibility and compatibility with soft robots; however, liquid metals can be difficult to handle and will add weight and bulk to a robot or sensor. Finally, liquid metals such as galinstan and eGaIn, a eutectic mixture of gallium and indium, create increased safety concerns as they are not biocompatible. ${ }^{84}$

Exteroceptive sensors. Exteroceptive sensors may be cameras, force sensors, or contact sensors and it is this class of sensor that could provide benefits to the surgeon such as force feedback or positioning and orientation information relative to the surrounding tissue. In the literature search results, there were only two examples of integrated exteroceptive sensing. One of these, a cable-driven, continuum robot designed for cardiac ablation, was manufactured with four optical fibers integrated into its elastomer body, which could measure the distance of the tip to a beating heart phantom. ${ }^{19}$ This proximity sensor was shown to reduce the error between the desired and actual distance from the distal end of the robot to the beating heart model, thereby facilitating the ablation procedure.

A method for estimating the normal and shear forces exerted on a hydraulically actuated STIFF-FLOP manipulator ${ }^{18}$ by developing a nonlinear finite element model to relate tip deflection and internal pressure change is given in Lindenroth et al. ${ }^{23}$ Maximum normal force errors of between 0.2 and $0.4 \mathrm{~N}$ for bending angles between $0^{\circ}$ and $60^{\circ}$ were reported, while maximum shear force error was dependent on the shear load itself and the tip deflection, with a maximum error of $0.316 \mathrm{~N}$ for low tip angles and a load of $1.5 \mathrm{~N}$. The hardware used for pressure measurement does not introduce rigid components to the manipulator, so the robot can still be deemed soft. When large shear forces were exerted on the robot, the force estimation errors increased; hence, further research is needed to develop more robust force sensing abilities. Developing a soft approach to force sensing that avoids bulky hardware is a promising target for research, which could provide soft robot operators with the sense of touch in the future.

Diagnostic sensors. A number of devices described in articles from the literature search included integrated cameras that would allow visual inspection of the immediate surroundings; however, only one device featured dedicated diagnostic equipment. The device integrated photoresist material into a pneumatically actuated elastomer that was used as an optical waveguide for fluorescence imaging. ${ }^{30} \mathrm{The}$ fluorescence is detected at a given input pressure, which can then be related to the bending angle of the device. This may be impractical if the bending angle of the probe cannot be measured accurately due to interactions with an unstructured, dynamic environment where the probe may come into unintentional contact with its surroundings, resulting in an inaccurately measured location of fluorescence. The inability to measure the bending angle of the soft actuator accurately again highlights the need for proprioceptive, exteroceptive, and diagnostic sensor fusion.

Optical fibers can be integrated into soft robotic devices, so it would be interesting to see some more diagnostic applications of optical fibers in soft robots. This may include optical biopsy techniques such as diffuse reflectance spectroscopy, optical coherence tomography, and endomicroscopy.

\section{Discussion and Conclusion}

\section{Comparison}

There exists no consistent standard of comparison for soft robotic devices designed for minimally invasive procedures. Given the variety of devices and operating principles under consideration, this makes it very difficult to compare candidate devices and identify knowledge gaps, engineering challenges, and improvements. A standardized datasheet is proposed in Grioli et al. ${ }^{85}$ for use in defining variable stiffness actuators. The template suggests designers graphically define relationships such as stiffness, speed, and deflection versus torque and the 3D workspace, as well as giving operating data, including output power, nominal and maximum torque, speed, stiffness, elastic energy, and sensor descriptions. 
In total, a very detailed picture of a variable stiffness actuator can be built.

However, it can be problematic to attempt to describe a device using only limited measurements, especially when the measured quantities are dependent upon each other. This is the case with some pneumatically actuated devices that have incorporated stiffening mechanisms, so the bending angle, force exertion, and stiffness are all interrelated. The pose of some soft robotic devices can also affect their mechanical properties, so it is indeed challenging to meaningfully describe these devices only in numbers. Furthermore, the proposed datasheet is for continuum-style actuators, so other robotic devices cannot be well compared, although the datasheet is quite comprehensive.

Instead, a focus on the capability of the proposed device to carry out a simplified, standardized version of the application it was designed for could be more useful and could make the advantages of the design clearer. An "assault course" of standard tasks, each related to a specific application, that all new devices could be tested on would provide metrics on whether or not a device provides performance advantages in each area. This approach, coupled with a standard datasheet, would make comparison far simpler.

\section{Application and working principle}

Given that continuum robots experience issues balancing size, force exertion, and controllability, there are few examples of alternative approaches in the literature. Continuum and serial robots do appear to be a good choice of manipulator; however, these robots also struggle with access to the target anatomy. Self-propelled devices with integrated continuum or serial manipulators may be the best way to improve on these issues. Providing a mobile base for robotic manipulators will require the development of variable stiffness mechanisms that can withstand higher forces; reliable selfpropulsion methods that can both achieve higher speeds and remain minimally invasive; and anchoring methods so that higher forces can be exerted by the device when it is in situ.

Designers could also take advantage of soft robotic devices' ability to change shape, volume, and even working principle online. Potential applications of this could be a soft robot to perform NOTES that can move through the GI tract using one locomotion strategy, and then change shape to use a different locomotion strategy in the abdominal cavity.

New soft robotic devices should aim at integrating soft proprioceptive, exteroceptive, and diagnostic sensors. The results of the literature search yielded very few examples of diagnostic sensors and many less well-developed devices did not clearly state what benefits they would deliver to surgeons in terms of what instruments could be used, if any channels were available. Furthermore, few devices described what the effect of having different instruments in place would be on controllability or force exertion. Traditional endoscopes provide a platform to quickly transition from diagnostic to therapeutic operation, for example, during a colonoscopy, a traditional endoscope can be used to not only image the colon but to also perform surgical procedures, although limited. Soft robotic devices for minimally invasive procedures should therefore also aim to provide both diagnostic and therapeutic capabilities. The advantages of traditional endoscopes can be expanded upon and the therapeutic capabilities improved by attaching deployable structures to the endoscope tip, such as the inflatable structure in Vrielink et al., ${ }^{86}$ which supports two cable-driven surgical instruments for intuitive, bimanual control.

As can be seen from the devices found in this study, very few soft robots for minimally invasive applications are made to be deployable. Deployability in this context is the capability to unfold or change shape to transition from a low to high volume, and in medical applications could be very useful for gaining access deeper into the body without the need for incisions to the abdominal cavity. This indicates that there are still important challenges to be overcome relating to the scalability of actuation mechanisms and the robots themselves, and that the material choices of the designers have not permitted deployability.

Almost all the devices require a tether and, in the context of MIS or colonoscopy where there is limited working space, this seems to be a reasoned design choice if the total footprint of the hardware is not extensive. Conversely, the long-term goal for the future should be the development of miniaturized, untethered, soft robots able to achieve unprecedented access throughout the body, causing no damage as they operate. Much work has been done in the field of capsule endoscopy to miniaturize vision and locomotion systems and incorporate them in individual untethered devices. As challenges such as achieving reliable and accurate control and achieving sufficient force exertion from miniaturized actuators are solved, the integration of approaches from capsule endoscopy and the field of soft robotics will become possible.

A soft robotic device is described in Wehner et al., ${ }^{87}$ which can operate autonomously using pneumatic elastomeric actuators powered by on-board chemical decomposition. Questions over the safety of using devices that rely upon chemical reactions in medical applications have been raised, but devices of this nature may become feasible for use in minimally invasive procedures if safety issues can be solved. Furthermore, the question of how much autonomy to give robotics devices within the body will need to be addressed.

\section{Materials and manufacture}

There is very little variety in the materials used in the soft robots encountered by the literature search. Very few designs use films of inextensible materials to make chambers for fluidic actuation and this may be because there does not currently exist a simple manufacturing method that uses these materials, in contrast to elastomers that can be processed by simple molding procedures.

Researchers in this area should explore a larger range of materials, although this may induce higher cost. Maintaining high performance at low cost is the key to making surgical technology accessible. Improved and accessible tools will enable surgeons with less experience to perform more challenging procedures, while low-cost solutions will prevent improved robotic systems from becoming exclusive to certain locations.

A new manufacturing approach would help to overcome the problems currently faced by soft robotics in MIS. Designers of soft robotic devices for MIS should consider manufacturing techniques that are low cost, fast, and capable of producing small features. High repeatability and reliability 
are also necessary, as well as being able to adapt both the designs and the manufacturing process. High adaptability also applies to being able to add or take away device functionality, for example, sensors or locomotion mechanisms, depending on what is required from the surgical procedure. Design adaptability and economical consumables will also enable disposable patient-specific soft robotics, which may reduce complications.

There may be certification issues regarding patient-specific devices and their route to obtaining CE marking, for example. As part of The Medical Devices Regulations 2002 legislation made by the UK government, a custom-made device is one that is made for the sole use of a particular patient. This legislation states that no custom-made device can be marketed unless the manufacturer can ensure the manufacturing process is capable of producing each individual device up to the given standard for that type of device. This shows the importance of developing devices using highly repeatable, reliable manufacturing processes. This could be problematic for devices that rely on molding processes that are sensitive to manufacturing defects such as bubbling or nonuniform thickness.

\section{Actuation}

None of the actuation methods mentioned are reversible so, for example, a cable or pneumatic actuator can only push or pull, but cannot do both. Using actuators that can only exert forces in one direction has the consequence that creative ways of exploiting material characteristics, combinations of actuators, and antagonistic arrangements of actuators are necessary. This requires more space and increases device complexity, which drives up manufacturing times, costs, and creates a conflict with competing factors such as the need for instrumentation channels.

The demand for actuation methods that are powerful, fast, lightweight, and low volume is also left unmet currently, but this may also be related to the design choices being made. Soft parallel mechanisms have not been explored and may provide improvements on the force exertion and controllability issues common to continuum designs. Manufacturing methods that can produce pressure chambers capable of withstanding much higher actuation pressures would deliver an increase in force exertion, and using both inextensible and elastomeric materials might result in higher maximum rigidity or larger ranges for variable rigidity methods. Difficulty modeling nonlinear systems such as pneumatic chambers and soft elastomer bodies also hinders the ability to design devices that can be controlled predictably.

\section{Acknowledgments}

This article is independent research funded by the National Institute for Health Research (NIHR) Imperial Biomedical Research Centre (BRC). The views expressed in this publication are those of the author(s) and not necessarily those of the NHS, the NIHR, or the Department of Health and Social Care.

\section{Author Disclosure Statement}

No competing financial interests exist.

\section{References}

1. Burgner-Kahrs J, Rucker DC, Choset H. Continuum robots for medical applications: A survey. IEEE Trans Robot 2015;31:1261-1280.

2. Kim HJ, Choi GS, Park JS, et al. Comparison of surgical skills in laparoscopic and robotic tasks between experienced surgeons and novices in laparoscopic surgery: An experimental study. Ann Coloproctol 2014;30:71-76.

3. Baron TH. Natural orifice transluminal endoscopic surgery. Br J Surg 2007;94:1-2.

4. Kassam AB, Engh JA, Mintz AH, et al. Completely endoscopic resection of intraparenchymal brain tumors. J Neurosurg 2009;110:116-123.

5. Anderson PL, Lathrop RA, Webster RJ. Robot-like dexterity without computers and motors: A review of handheld laparoscopic instruments with wrist-like tip articulation. Expert Rev Med Devices 2016;13:661-672.

6. Picod G, Jambon AC, Vinatier D, et al. What can the operator actually feel when performing a laparoscopy?. Surg Endosc Other Interv Tech 2005;19:95-100.

7. Rané A, Tan GY, Tewari AK. Laparo-endoscopic singlesite surgery in urology: Is robotics the missing link?. Br J Urol Int 2009; 104:1041-1043.

8. Vitiello V, Lee SL, Cundy TP, et al. Emerging robotic platforms for minimally invasive surgery. IEEE Rev Biomed Eng 2013;6:111-126.

9. Loeve A, Breedveld P, Dankelman J. Scopes too flexible and too stiff. IEEE Pulse 2010;1:26-41.

10. Shah SG, Saunders BP, Brooker JC, et al. Magnetic imaging of colonoscopy: An audit of looping, accuracy and ancillary maneuvers. Gastrointest Endosc 2000;52:1-8.

11. Voermans RP, Van Berge Henegouwen MI, et al. Natural orifice transluminal endoscopic surgery. Endoscopy 2007; 39:1013-1017.

12. Laschi C, Cianchetti M. Soft robotics: New perspectives for robot bodyware and control. Front Bioeng Biotechnol 2014;2:1-5.

13. Kim S, Laschi C, Trimmer B. Soft robotics: A bioinspired evolution in robotics. Trends Biotechnol 2013;31:287-294.

14. Cianchetti M, Laschi C, Menciassi A, et al. Biomedical applications of soft robotics. Nat Rev Mater 2018;3:1-11.

15. Hughes J, Culha U, Giardina F, et al. Soft manipulators and grippers: A review. Front Robot AI 2016;3:1-12.

16. Lipson H. Challenges and opportunities for design, simulation, and fabrication of soft robots. Soft Robot 2014;1: 21-27.

17. Majidi C. Soft robotics: A perspective-Current trends and prospects for the future. Soft Robot 2014;1:5-11.

18. Cianchetti M, Ranzani T, Gerboni G, et al. STIFF-FLOP surgical manipulator: Mechanical design and experimental characterization of the single module. IEEE Int Conf Intell Robot Syst 2013;3576-3581.

19. Liu Y, Xie H, Wang H, et al. Distance control of soft robot using proximity sensor for beating heart surgery. In: 2016 IEEE/SICE International Symposium on System Integration (SII), held at Sapporo, December 13-15, 2016:403408.

20. Wang H, Zhang R, Chen W, et al. A cable-driven soft robot surgical system for cardiothoracic endoscopic surgery: Preclinical tests in animals. Surg Endosc Other Interv Tech 2017;31:3152-3158.

21. Kim YJ, Cheng S, Kim S, et al. A novel layer jamming mechanism with tunable stiffness capability for minimally invasive surgery. IEEE Trans Robot 2013;29:1031-1042. 
22. Stilli A, Wurdemann HA, Althoefer K. Shrinkable, stiffness-controllable soft manipulator based on a bioinspired antagonistic actuation principle. In: IEEE/RSJ International Conference on Intelligent Robots and Systems, Chicago, IL, September 14-18, 2014. pp. 24762481.

23. Lindenroth L, Duriez $\mathrm{C}$, Back $\mathrm{J}$, et al. Intrinsic force sensing capabilities in compliant robots comprising hydraulic actuation. IEEE Int Conf Intell Robot Syst 2017; 2923-2928.

24. Stopforth R, Davrajh S, Althoefer K. Low cost soft endoscope robotic probe. In: 2017 IEEE AFRICON, Cape Town, September 18-20, 2017. pp. 1414-1419.

25. Surakusumah RF, Faudzi AAM, Dewi DEO, et al. Development of a half sphere bending soft actuator for flexible bronchoscope movement. 2014 IEEE Int Symp Robot Manuf Autom IEEE ROMA 2015:120-125.

26. Gorissen B, De Volder M, Reynaerts D. Chip-on-tip endoscope incorporating a soft robotic pneumatic bending microactuator. Biomed Microdevices 2018;20:1-7.

27. Yan J, Dong H, Zhang X, et al. A three-chambed soft actuator module with omnidirectional bending motion. In 2016 IEEE International Conference on Real-Time Computing and Robotics (IEEE RCAR) 2016:505-510.

28. Sun Y, Song S, Liang X, et al. A miniature soft robotic manipulator based on novel fabrication methods. IEEE Robot Autom Lett 2016;1:617-623.

29. Lee KH, Fu DKC, Leong MCW, et al. Nonparametric online learning control for soft continuum robot: An enabling technique for effective endoscopic navigation. Soft Robot 2017;4:324-337.

30. Muramatsu Y, Kobayashi T, Konishi S. Flexible endeffector integrated with scanning actuator and optical waveguide for endoscopic fluorescence imaging diagnosis. Proc IEEE Int Conf Micro Electro Mech Syst 2015:166167.

31. Gorissen B, Vincentie W, Al-Bender F, et al. Modeling and bonding-free fabrication of flexible fluidic microactuators with a bending motion. J Micromech Microeng 2013;23:045012.

32. Elsayed Y, Vincensi A, Lekakou C, et al. Finite element analysis and design optimization of a pneumatically actuating silicone module for robotic surgery applications. Soft Robot 2014;1:255-262.

33. Rehman T, Faudzi AAM, Dewi DEO, et al. Design, characterization, and manufacturing of circular bellows pneumatic soft actuator. Int J Adv Manuf Technol 2017;93: 4295-4304.

34. Liang X, Sun Y, Ren H. A flexible fabrication approach toward the shape engineering of microscale soft pneumatic actuators. IEEE Robot Autom Lett 2016;2:165-170.

35. Li Y, Chen Y, Yang Y, et al. Passive particle jamming and its stiffening of soft robotic grippers. IEEE Trans Robot 2017;33:446-455.

36. Feng TZ, Ren H. Towards disposable and printable robotic endoscopic surgery system. In 2014 11th World Congress on Intelligent Control and Automation (WCICA) 2014: 992-996.

37. Shim JE, Quan YJ, Wang W, et al. A smart soft actuator using a single shape memory alloy for twisting actuation. Smart Mater Struct 2015;24:125033.

38. Tan N, Gu X, Ren H. Design, characterization and applications of a novel soft actuator driven by flexible shafts. Mech Mach Theory 2018;122:197-218.
39. Shiva A, Stilli A, Noh Y. Tendon-based stiffening for a pneumatically actuated soft manipulator. IEEE Robot Autom Lett 2016;1:632-637.

40. Ikuta K, Matsuda Y, Yajima D, et al. Pressure pulse drive: A control method for the precise bending of hydraulic active catheters. IEEE ASME Trans Mechatronics 2012;17: 876-883.

41. Abidi H, Gerboni G, Brancadoro M, et al. Highly dexterous 2-module soft robot for intra-organ navigation in minimally invasive surgery. Int J Med Robot Comput Assist Surg 2018;14:1-9.

42. Arezzo A, Mintz Y, Allaix ME, et al. Total mesorectal excision using a soft and flexible robotic arm: A feasibility study in cadaver models. Surg Endosc Other Interv Tech 2017;31:264-273.

43. Gerboni G, Ranzani T, Diodato A, et al. Modular soft mechatronic manipulator for minimally invasive surgery (MIS): Overall architecture and development of a fully integrated soft module. Meccanica 2015;50:28652878.

44. Diodato A, Brancadoro M, De Rossi G, et al. Soft robotic manipulator for improving dexterity in minimally invasive surgery. Surg Innov 2018;25:69-76.

45. Cianchetti M, Ranzani T, Gerboni G, et al. Soft robotics technologies to address shortcomings in today's minimally invasive surgery: The STIFF-FLOP approach. Soft Robot 2014;1:122-131.

46. Bernth JE, Arezzo A, Liu H. A novel robotic meshworm with segment-bending anchoring for colonoscopy. IEEE Robot Autom Lett 2017;2:1.

47. Haga Y, Mineta $\mathrm{T}$, Makishi $\mathrm{W}$, et al. Active bending catheter and endoscope using shape memory alloy actuators. Cdn.Intechweb.Org 1998:107-127.

48. Low JH, Delgado-Martinez I, Yeow CH. Customizable soft pneumatic chamber-gripper devices for delicate surgical manipulation. J Med Device 2014;8:044504.

49. $\mathrm{Hu} \mathrm{W}$, Lum GZ, Mastrangeli M, et al. Small-scale softbodied robot with multimodal locomotion. Nature 2018; 554:81-85.

50. Pacchierotti C, Ongaro F, van den Brink F, et al. Steering and control of miniaturized untethered soft magnetic grippers with haptic assistance. IEEE Trans Autom Sci Eng 2018;15:290-306.

51. Suzumori K, Hama T, Kanda T. New pneumatic rubber actuators to assist colonoscope insertion. Proc IEEE Int Conf Robot Autom 2006;1824-1829.

52. Sadati SMH, Naghibi SE, Shiva A, et al. A geometry deformation model for braided continuum manipulators. Front Robot AI 2017;4:1-25.

53. Connolly F, Polygerinos $\mathrm{P}$, Walsh CJ, et al. Mechanical programming of soft actuators by varying fiber angle. Soft Robot 2015;2:26-32.

54. Calisti M, Picardi G, Laschi C. Fundamentals of soft robot locomotion. J R Soc Interface 2017;14:20170101.

55. Takeshima H, Takayama T. Geometric estimation of the deformation and the design method for developing helical bundled-tube locomotive devices. IEEE ASME Trans Mechatronics 2018;23:223-232.

56. Kim HJ, Kawamura A, Nishioka Y, et al. Mechanical design and control of inflatable robotic arms for high positioning accuracy. Adv Robotics 2018;32:89-104.

57. Niiyama R, Rus D, Kim S. Pouch motors: Printable/ inflatable soft actuators for robotics. Proc IEEE Int Conf Robot Autom 2014;6332-6337. 
58. Russo S, Ranzani T, Gafford J, et al. Soft pop-up mechanisms for micro surgical tools: Design and characterization of compliant millimeter-scale articulated structures. In 2016 IEEE International Conference on Robotics and Automation (ICRA), 2016:750-757.

59. Li Z, Du R. Design and analysis of a bio-inspired wiredriven multi-section flexible robot. Int $\mathrm{J}$ Adv Robot Syst 2013;10:209-219.

60. Li Z, Feiling J, Ren H, et al. A novel tele-operated flexible robot targeted for minimally invasive robotic surgery. Engineering 2015;1:73-78.

61. Polygerinos P, Correll N, Morin SA, et al. Soft robotics: Review of fluid-driven intrinsically soft devices; manufacturing, sensing, control, and applications in human-robot interaction. Adv Eng Mater 2017;19:1700016.

62. Gilbertson MD, McDonald G, Korinek G, et al. Soft passive valves for serial actuation in a soft hydraulic robotic catheter. J Med Device 2016;10:030931.

63. Zatopa A, Walker S, Menguc Y. Fully soft 3D-printed electroactive fluidic valve for soft hydraulic robots. Soft Robot 2018;5:258-271.

64. Morimoto TK, Okamura AM. 3D printed concentric tube robot design. IEEE Trans Robot vol Conditiona 2016:1-12.

65. Pourghodrat A, Nelson CA. Disposable fluidic actuators for miniature in-vivo surgical robotics. J Med Device 2014;8: 030920 .

66. Gorissen B, Reynaerts D, Konishi S, et al. Elastic inflatable actuators for soft robotic applications. Adv Mater 2017;29: $1-14$.

67. Connolly F, Polygerinos $\mathrm{P}$, Walsh CJ, et al. Mechanical programming of soft actuators by varying fiber angle. Soft Robot 2015;2:26-32.

68. Ou J, Skouras M, Vlavianos N, et al. aeroMorph-Heatsealing inflatable shape-change materials for interaction design. In Proceedings of the 29th Annual Symposium on User Interface Software and Technology-UIST'16 2016: 121-132.

69. Xuan Y, Shuai C, Gao Y, et al. Application review of dielectric electroactive polymers (DEAPs) and piezoelectric materials for vibration energy harvesting. J Phys Conf Ser 2016;744:012077.

70. De Luca V, Digiamberardino P, Di Pasquale G et al. Ionic electroactive polymer metal composites: Fabricating, modeling, and applications of postsilicon smart devices. J Polym Sci Part B Polym Phys 2013;51:699-734.

71. Blanc L, Delchambre A, Lambert P. Flexible medical devices: Review of controllable stiffness solutions. Actuators 2017;6:23.

72. Narang YS, Vlassak JJ, Howe RD. Mechanically versatile soft machines through laminar jamming. Adv Funct Mater 2018;1707136:1-9.

73. Witte TN, Enns R. The difficult colonoscopy. Can J Gastroenterol 2007;21:487-490.

74. Alazmani A, Hood A, Jayne D, et al. Quantitative assessment of colorectal morphology: Implications for robotic colonoscopy. Med Eng Phys 2016;38:148-154.
75. Valdastri P, Webster RJ, Quaglia C, et al. A new mechanism for mesoscale legged locomotion in compliant tubular environments. IEEE Trans Robot 2009;25:1047-1057.

76. Dickey MD. Stretchable and soft electronics using liquid metals. Adv Mater 2017;29:1-19.

77. Liang X, Sun Y, Ren H. A flexible fabrication approach towards the shape engineering of microscale soft pneumatic actuators. IEEE Robot Autom Lett 2016;2:165-170.

78. Amjadi M, Kyung KU, Park I, et al. Stretchable, skinmountable, and wearable strain sensors and their potential applications: A review. Adv Funct Mater 2016;26:16781698.

79. Al Jaber F, Althoefer K. Towards creating a flexible shape senor for soft robots. 2018 IEEE Int Conf Soft Robot RoboSoft 2018:114-119.

80. Wang H, Zhang R, Chen W, et al. Shape detection algorithm for soft manipulator based on fiber bragg gratings. IEEE ASME Trans Mechatronics 2016;21:2977-2982.

81. Shi C, Luo X, Qi P. Shape sensing techniques for continuum robots in minimally invasive surgery: A survey. IEEE Trans Biomed Eng 2017;64:1665-1678.

82. Ho JDL, Lee KH, Tang WL, et al. Localized online learning-based control of a soft redundant manipulator under variable loading. Adv Robotics 2018;32:1-16.

83. Zhou LY, Gao Q, Zhan JF, et al. Three-dimensional printed wearable sensors with liquid metals for detecting the pose of snakelike soft robots. ACS Appl Mater Interfaces 2018; 10:23208-23217.

84. Russo S, Ranzani T, Liu H, et al. Soft and stretchable sensor using biocompatible electrodes and liquid for medical applications. Soft Robot 2015;2:146-154.

85. Grioli G, Wolf S, Garabini M, et al. Variable stiffness actuators: The user's point of view. Int J Rob Res 2015;34: 727-743.

86. Vrielink TJCO, Zhao M, Darzi A, et al. ESD CYCLOPS: A new robotic surgical system for GI surgery. 2018 IEEE Int Conf Robot Autom 2017;150-157.

87. Wehner M, Truby RL, Fitzgerald DL, et al. An integrated design and fabrication strategy for entirely soft, autonomous robots. Nature 2016;536:451-455.

88. De Falco I, Cianchetti M, Menciassi A. A soft multi-module manipulator with variable stiffness for minimally invasive surgery. Bioinspir Biomim 2017;12:056008.

Address correspondence to:

Mark Runciman

Human-Centred Automation, Robotics and Monitoring in Surgery (HARMS) Lab Department of Surgery and Cancer Imperial College London London W2 IPE United Kingdom

E-mail: m.runciman@imperial.ac.uk 